\title{
Renal Effects of Dapagliflozin in People with and without Diabetes with Moderate or Severe Renal Dysfunction: Prospective Modeling of an Ongoing Clinical Trial[
}

\author{
K. Melissa Hallow, David W. Boulton, Robert C. Penland, Gabriel Helmlinger, \\ Emily H. Nieves, Daniël H. van Raalte, Hiddo L. Heerspink, and Peter J. Greasley \\ Department of Chemical, Materials, and Biomedical Engineering, University of Georgia, Athens, Georgia (K.M.W., E.N.); Clinical \\ Pharmacology and Quantitative Pharmacology, Clinical Pharmacology and Safety Sciences, R\&D, AstraZeneca, Gaithersburg, \\ Maryland (D.W.B.); Clinical Pharmacology and Quantitative Pharmacology, Clinical Pharmacology and Safety Sciences, R\&D, \\ AstraZeneca, Waltham, Massachusetts (R.C.P., G.H.); Diabetes Center, Department of Internal Medicine, Amsterdam University \\ Medical Centers, location VUMC, Amsterdam, The Netherlands (D.H.v.R.); Department of Clinical Pharmacy and Pharmacology, \\ University of Groningen, Groningen, Netherlands (H.L.H.); The George Institute for Global Health, Sydney, Australia (H.L.H.); and \\ Early Clinical Development, Research and Early Development, Cardiovascular, Renal and Metabolism (CVRM) \\ BioPharmaceuticals R\&D, AstraZeneca, Gothenburg, Sweden (P.J.G.)
}

Received March 27, 2020; accepted June 9, 2020

\section{ABSTRACT}

Sodium glucose cotransporter 2 inhibitors (SGLT2i) reduce cardiovascular events and onset and progression of renal disease by mechanisms that remain incompletely understood but may include clearance of interstitial congestion and reduced glomerular hydrostatic pressure. The ongoing DAPASALT mechanistic clinical study will evaluate natriuretic, diuretic, plasma/extracellular volume, and blood pressure responses to dapagliflozin in people with type 2 diabetes with normal or impaired renal function (D-PRF and D-IRF, respectively) and in normoglycemic individuals with renal impairment (N-IRF). In this study, a mathematical model of renal physiology, pathophysiology, and pharmacology was used to prospectively predict changes in sodium excretion, blood and interstitial fluid volume (IFV), blood pressure, glomerular filtration rate, and albuminuria in DAPASALT. After validating the model with previous diabetic nephropathy trials, virtual patients were matched to DAPASALT inclusion/exclusion criteria, and the DAPASALT protocol was simulated. Predicted changes in glycosuria, blood pressure, glomerular filtration rate, and albuminuria were consistent with other recent studies in similar populations. Predicted albuminuria reductions were $46 \%$ in D-PRF, $34.8 \%$ in D-IRF, and $14.2 \%$ in $\mathrm{N}$-IRF. The model predicts a similarly large IFV reduction between D-PRF and D-IRF and less, but still substantial, IFV reduction in N-IRF, even though glycosuria is attenuated in groups with impaired renal function. When DAPASALT results become available, comparison with these simulations will provide a basis for evaluating how well we understand the cardiorenal mechanism(s) of SGLT2i. Meanwhile, these simulations link dapagliflozin's renal mechanisms to changes in IFV and renal biomarkers, suggesting that these benefits may extend to those with impaired renal function and individuals without diabetes.

\section{SIGNIFICANCE STATEMENT}

Mechanisms of SGLT2 inhibitors' cardiorenal benefits remain incompletely understood. We used a mathematical model of renal physiology/pharmacology to prospectively predict responses to dapagliflozin in the ongoing DAPASALT study. Key predictions include similarly large interstitial fluid volume (IFV) reductions between subjects with normal and impaired renal function and less, but still substantial, IFV reduction in those without diabetes, even though glycosuria is attenuated in these groups. Comparing prospective simulations and study results will assess how well we understand the cardiorenal mechanism(s) of SGLT2 inhibitors.

\section{Introduction}

Sodium glucose cotransporter 2 inhibitors (SGLT2i) have been shown to reduce cardiovascular (and particularly heart failure) events and improve renal outcomes in people with type 2 diabetes (T2D) (Zinman et al., 2015; Mosenzon et al., 2019). Although SGLT2 inhibition produces an initial hemodynamic drop in GFR, results from EMPA-REG, CANVAS, and DECLARE outcomes trials demonstrated that kidney

This study was funded by AstraZeneca Pharmaceuticals. https://doi.org/10.1124/jpet.120.000040.

S This article has supplemental material available at jpet.aspetjournals.org. function in the treated groups stabilized, whereas the placebo group progressed (Wanner et al., 2016; Guthrie, 2018; Mosenzon et al., 2019). Post hoc analyses of phase III studies have found that dapagliflozin stabilized estimated GFR (eGFR) decline for up to 2 years (Fioretto et al., 2015) and reduced urinary albumin creatinine ratio (UACR) by $38 \%-48 \%$ in those with elevated albuminuria at baseline (Dekkers et al., 2018). Empagliflozin reduced the risk of new onset of macroalbuminuria, doubling of serum creatinine, and initiation of dialysis treatment, respectively (Wanner et al., 2016).

The mechanisms responsible for these cardiovascular and renoprotective effects remain incompletely understood. 
Renoprotective mechanisms may include reduced glomerular hydrostatic pressure, reduced proximal tubule sodium transport both directly and through coupled NHE3 inhibition, and/ or reduced blood pressure (Hallow et al., 2018a). In addition, sodium and glucose excretion with SGLT2i induces an osmotic diuresis, which could be responsible for improved heart failure outcomes (Hallow et al., 2018b).

Mathematical modeling provides a tool to describe, test, and quantitatively evaluate proposed mechanisms by which SGLT2 inhibition impacts renal and cardiovascular function. We have previously modeled the renal effects of dapagliflozin and identified a set of mechanisms capable of reproducing urinary and plasma biomarker responses observed in healthy subjects (Hallow et al., 2018a,b). Simulations with this model have demonstrated mathematically that SGLT2i reduces glomerular hydrostatic pressure as an indirect consequence of reduced proximal tubule sodium reabsorption (Vallon and Thomson, 2017). This provides a plausible explanation for the reduction in albuminuria and slowing of renal progression observed with SGLT2i. In addition, simulations predicted that SGLT2 inhibition will reduce interstitial fluid volume to a greater extent than blood volume compared with other forms of natriuretic/diuretic agents (Hallow et al., 2018a; Mahato et al., 2018). This suggests that in states of volume overload, such as heart failure, SGLT2 inhibition may relieve interstitial congestion without excessive lowering of blood volume and blood pressure, thus maintaining organ perfusion and possibly also preventing excessive neurohormonal activation.

Although SGLT2 inhibition has been shown to reduce total body fluid volume, no study has yet distinguished the relative effects of SGLT2 inhibition on blood and interstitial fluid volume during standardized sodium intake. The DAPASALT study (NCT03152084) is an open label, phase IV, three-arm mechanistic study designed to evaluate the natriuretic, diuretic, and blood pressure responses to 2 weeks of dapagliflozin treatment in people with $\mathrm{T} 2 \mathrm{D}$ with and without renal impairment and in normoglycemic individuals with renal impairment. Data obtained from this study may allow clinical evaluation of model-based mechanistic hypotheses, including the relatively larger effect on interstitial fluid volume compared with blood volume. The true test of any mathematical model is its ability to prospectively predict behavior. In this analysis, we extend our existing model to prospectively simulate changes in urinary clinical chemistry variables, blood volume, interstitial fluid volume, GFR, and urinary albumin excretion rate (UAER) in the ongoing mechanistic clinical DAPASALT study. This will evaluate the extent to which we truly understand the renal mechanisms of SGLT2i and may also identify gaps in our existing knowledge.

\section{Methods}

\section{Modeling Approach Overview}

Using a previously developed mathematical model of renal function and diabetic kidney disease (Hallow et al., 2014, 2017, 2018a; Mahato et al., 2018), we generated a population of virtual patients with diabetes and varying degrees of kidney injury by varying model parameters associated with T2D, hypertension (a common comorbidity with diabetes), and kidney injury. Because the effects of T2D on kidney injury in the model were previously developed based on data from $\mathrm{db} / \mathrm{db}$ mice with or without uninephrectomy (Mahato et al., 2018), we used human diabetic nephropathy clinical trial data to recalibrate rate constants for this component of the model and to validate the simulated response to standard-of-care therapies [i.e., ACE inhibitors (ACEI) and angiotensin receptor blockers (ARBs)]. We then selected a population of virtual patients to match the DAPASALT inclusion/exclusion criteria and simulated the protocol of the ongoing DAPASALT study.

\section{Model Description}

The model of renal function and diabetic kidney injury is summarized in Fig. 1 and has been described in detail previously (Hallow and Gebremichael, 2017a,b; Hallow et al., 2018a; Mahato et al., 2018). This model describes the key physiologic processes of renal function and their roles in maintaining $\mathrm{Na}^{+}$and water homeostasis, as well as pathologic processes leading to renal injury and proteinuria in diabetes. Full model equations are also provided in the Supplemental Material. Here, we provide an overview of the model and describe only key model equations necessary to understand how renal injury and albuminuria were modeled, how parameters varied to generate virtual patients, and how SGLT2 inhibition was modeled.

Renal Vasculature. As shown in Fig. 1 (top left), the kidney is modeled as a set of nephrons in parallel.

Renal blood flow is a function of the mean arterial pressure (MAP), renal venous pressure, and renal vascular resistance, according to Ohm's law (Supplemental eqs. A1-A4). Renal vascular resistance is the equivalent resistance of preafferent, afferent, efferent, and peritubular arterioles and capillaries, and it also depends on the number of nephrons.

Glomerular Filtration. Single nephron glomerular filtration rate (SNGFR) depends on the glomerular ultrafiltration coefficient $\left(\mathrm{K}_{\mathrm{f}}\right)$ as well as the net filtration pressure across the glomerulus, according to Starling's equation:

$$
S N G F R=K_{f}\left(P_{g c}-P_{B o w}-\pi_{g o-a v g}\right)
$$

Here, $K_{f}$ is the glomerular ultrafiltration coefficient, $P_{g c}$ is glomerular capillary hydrostatic pressure, $P_{B o w}$ is pressure in the Bowman's space, and $\pi_{\text {go-avg }}$ is average glomerular capillary oncotic pressure. The total GFR is then the SNGFR multiplied by the number of nephrons $\left(N_{\text {nephrons }}\right)$ :

$$
G F R=S N G F R * N_{\text {nephrons }}
$$

\footnotetext{
ABBREVIATIONS: ACE, angiotensin converting enzyme; ACEI, ACE inhibitor; Ang, angiotensin; ARB, angiotensin receptor blocker; AT1, Angiotensin Type 1; AT2, Angiotensin Type 2; CANAVAS, CANagliflozin cardioVascular Assessment Study; AVOID, Aliskiren in the Evaluation of Proteinuria in Diabetes; $\mathrm{C}_{\text {glu }}$, plasma glucose concentration; CKD, chronic kidney disease; D-IRF, patients with type 2 diabetes with impaired renal function; D-PRF, patients with type 2 diabetes with preserved renal function; eGFR, estimated GFR; EMPAREG, Empagliflozin Cardiovascular Outcome Event Trial in Type 2 Diabetes Mellitus Patients; GFR, glomerular filtration rate; IDNT, Irbesartan Diabetic Nephropathy Trial; IFV, interstitial fluid volume; MAP, mean arterial pressure; NESTOR, Natrilix SR versus Enalapril Study in Type 2 diabetic hypertensives with micrOalbuminuRia;

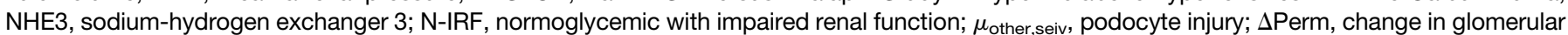
membrane permeability; $\mathrm{P}_{\mathrm{gc}}$, glomerular capillary hydrostatic pressure; PT, proximal tubule; RAAS, renin-angiotensin-aldosterone system; RENAAL, Reduction of Endpoints in NIDDM with the Angiotensin II Antagonist Losartan Study; $\mathrm{RC}_{\text {albumin, }}$, proximal tubule capacity to reabsorb a filtered albumin; SGLT, sodium glucose cotransporter; SGLT2i, SGLT2 inhibitor; SNGFR, single nephron glomerular filtration rate; T2D, type 2 diabetes; UACR, urinary albumin creatinine ratio; UAER, urinary albumin excretion rate; UGE, urinary glucose excretion.
} 


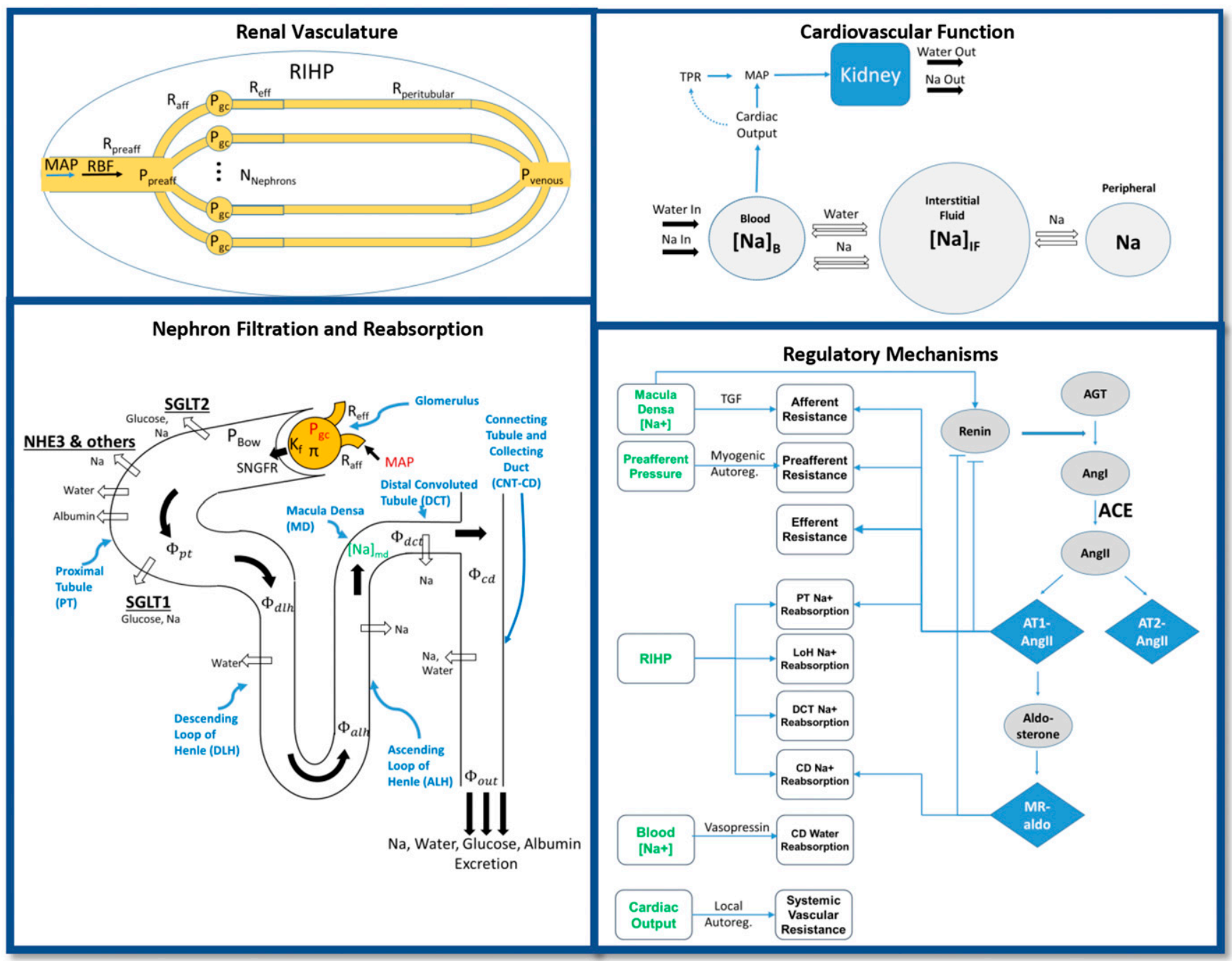

Fig. 1. Base model of renal function. (Top left) The renal vasculature is modeled by a single preafferent resistance vessel flowing into $\mathrm{N}$ parallel nephrons. (Bottom left) Glomerular filtration is modeled according to Starling's law. $\mathrm{Na}^{+}$and water are reabsorbed at different fractional rates in the proximal tubule, loop of Henle, distal convoluted tubule, and connecting tubule/collecting duct. Glucose and $\mathrm{Na}^{+}$reabsorption are coupled through SGLT2 and SGLT1 in the proximal tubule. (Top right) The balance between $\mathrm{Na}^{+}$and water excretion and intake determine blood volume and $\mathrm{Na}^{+}$concentration. $\mathrm{Na}^{+}$and water move between the blood and interstitial fluid across a concentration gradient, and $\mathrm{Na}^{+}$may be sequestered nonosmotically in a peripheral storage compartment. Blood volume determines venous return and cardiac output, which together with total peripheral resistance, determine mean arterial pressure and subsequently renal perfusion pressure, closing the loop. (Bottom right) Multiple regulatory mechanisms, including the RAAS and tubuloglomerular feedback (TGF), provide feedback on model variables to maintain or return homeostasis. $\Phi$, flow rate; CD, collecting duct; CNT-CD, connecting tubule and collecting duct; DLH, descending loop of Henle; $\mathrm{N}_{\mathrm{Nephron}}$, number of nephrons; $\mathrm{P}_{\mathrm{Bow}}$, pressure in the Bowman's space; $\mathrm{R}_{\mathrm{aff}}$, afferent arteriole resistance; $\mathrm{RBF}$, renal blood flow; RIHP, renal interstitial hydrostatic pressure set point; $\mathrm{R}_{\text {preaff, }}$ preafferent arteriole resistance; $\mathrm{P}_{\text {venous, }}$, venous pressure; LoH, loop of Henle; $\mathrm{R}_{\text {peritubular, }}$, peritubular capillary resistance; TPR, total peripheral resistance Adapted from Hallow et al. (2017).

Glucose Filtration, Reabsorption, and Excretion. Glucose is filtered freely through the glomerulus. Thus, the filtered load for a single nephron is the product of SNGFR and average plasma glucose concentration $\left(C_{\text {glu }}\right)$ :

$$
\Phi_{\text {glu filtered }}=S N G F R^{*} \mathrm{C}_{\text {glu }} .
$$

Glucose is reabsorbed by SGLT2 in the S1 and S2 segments of the proximal tubule (PT) and by SGLT1 in the S3 segment, up to the reabsorptive capacity of each segment (Supplemental eqs. A8 and A9). Any unreabsorbed glucose then flows through the rest of the tubule and is excreted in the urine (Supplemental eq. A10).

$\mathrm{Na}^{+}$Filtration and Reabsorption. The single nephron-filtered $\mathrm{Na}^{+}$load is given by:

$$
\Phi_{\mathrm{Na}, \text { filtered }}=S N G F R^{*} C_{N a}
$$

where $C_{N a}$ is the plasma $\mathrm{Na}^{+}$concentration. $\mathrm{Na}^{+}$is reabsorbed through different transporters at different rates in each segment along the tubule. In the proximal tubule, NHE3 plays a major role in $\mathrm{Na}^{+}$reabsorption, and thus NHE3 reabsorption is modeled explicitly. In addition, coupling of glucose and $\mathrm{Na}^{+}$reabsorption through SGLT2 at a 1:1 molar ratio (Supplemental eq. A12) and by SGLT1 at a 1:2 molar ratio (Supplemental eq. A13) is modeled. Additional $\mathrm{Na}^{+}$reabsorption through other transporters is also accounted for (Supplemental eq. A14). For the remaining nephron segments, we approximate $\mathrm{Na}^{+}$reabsorption in each segment as distributed uniformly along the length and defined by a fractional rate of reabsorption (Supplemental eqs. A15-A18).

Dapagliflozin treatment is not associated with changes in serum potassium, so for simplicity, potassium filtration and reabsorption were not tracked in the model (Yavin et al., 2016).

Water Reabsorption. Water reabsorption in the PT is isosmotic. Thus, the rate of water reabsorption depends on the concentration of osmolytes, including $\mathrm{Na}^{+}$and glucose, in the tubular fluid (Supplemental eqs. A19-A21). 
The flow rates of osmolytes and water out of the PT are then used to determine water reabsorption along the remaining nephron segments, including regulation by vasopressin in the collecting duct, as described previously and in the Supplemental eqs. A22-A28.

Blood and Interstitial Fluid and Peripheral Sodium Storage. Sodium and water are modeled as distributed between the blood, interstitium, and a third compartment that stores $\mathrm{Na}^{+}$ nonosmotically (Fig. 1 (top right)) (Titze, 2009, 2014; Hammon et al., 2015; Hallow et al., 2018b). Sodium and water are assumed to move freely between the blood and interstitial fluid across a $\mathrm{Na}^{+}$concentration gradient. Water and sodium intake rates are assumed constant. Then, blood volume and blood sodium are the balance between intake and excretion of water and sodium, respectively, and the intercompartmental transfer between blood and interstitium (Supplemental eqs. A29 and A30). Similarly, interstitial fluid volume (IFV) depends on the intercompartmental transfer between blood and interstitium (Supplemental eq. A31).

When interstitial sodium concentration exceeds the normal equilibrium level, $\mathrm{Na}^{+}$is assumed to move out of the interstitium and is sequestered in the peripheral $\mathrm{Na}^{+}$compartment, where it is osmotically inactive. Thus, the change in interstitial fluid sodium depends on intercompartmental transfer and peripheral storage (Supplemental eqs. A32-A34). Sodium cannot be stored indefinitely; thus, there is a limit on how much sodium can be stored.

Albumin Filtration, Reabsorption, and Excretion. The rate of albumin filtration is a function of SNGFR, the plasma albumin concentration $C_{\text {albumin }}$, and the sieving coefficient $K_{\text {albumin }}$, as described in Lazzara and Deen (2007):

$$
\Phi_{\text {albumin.filtered }}=K_{\text {albumin }} * \mathrm{SNGFR} * \mathrm{C}_{\text {albumin }}
$$

The PT has limited capacity to reabsorb a filtered albumin $\left(R C_{\text {albumin }}\right)$, beyond which excess albumin is excreted.

$$
\Phi_{\text {albumin }, \text { reabs }}=\min \left(\Phi_{\text {albumin,filtered }}, R C_{\text {albumin }}\right) .
$$

The UAER is then

$$
\text { UAER }=\left(\Phi_{\text {albumin filtered }}-\Phi_{\text {albumin,reabs }}\right) * \mathrm{~N}_{\text {nephrons }} .
$$

Kidney Injury. Nephron loss due to kidney injury was modeled by reducing the number of nephrons. Although nephron loss in kidney disease is progressive, we did not account for progressive nephron loss in the current analysis, since all simulation durations were less than 6 months.

We assumed that, when glomerular capillary hydrostatic pressure $P_{g c}$ rises above some normal limit $P_{g c, o}$, it causes injury and dysfunction of the glomerulus and podocytes. The magnitude of this injury signal $\left(\mathrm{GP}_{\text {njury }}\right)$ is defined as:

$$
G P_{\text {injury }}=\max \left(P_{g c}-P_{g c 0}, 0\right) .
$$

Glomerular hypertension causes glomerular hypertrophy, with up to a 50\% increase in glomerular volume observed within a few weeks in diabetic and/or nephrectomized rats, mice, and humans (Flyvbjerg et al., 2002; Levine et al., 2008; Bivona et al., 2011). The ultrafiltration coefficient $K_{f}$ in eq. 1 above reflects both the permeability and surface area of the glomerular membrane. The effect of glomerular hypertension on $K_{f}$ through changes in the glomerular surface area (hypertrophy) is modeled as

$$
\frac{d}{d t}(\Delta S A)=\left(\Delta S A_{\max }-\Delta S A\right) * \frac{\mathrm{GP}_{\text {injury }}}{\tau_{\mathrm{SA}}} .
$$

$\Delta S A_{\text {max }}$ is the maximum increase in glomerular surface area (SA; expressed as a percentage), and $\tau_{S A}$ represents the time constant for the increase in surface area. $\Delta S A_{\max }$ is fixed at $50 \%$, and the time constant $\tau_{S A}$ is set so that a steady state is reached within a few weeks.
Glomerular hypertension also contributes to progressive glomerulosclerosis, which is a slower process than glomerular hypertrophy. Mathematically, this can be represented as a decrease in glomerular permeability $\Delta$ Perm, so that the ultrafiltration coefficient $K_{f}$ is given by

$$
K_{f}=K_{f, 0} *(1+\Delta \mathrm{SA}-\Delta \text { Perm })
$$

where $K_{f, 0}$ is the normal ultrafiltration coefficient in the healthy state. For this analysis, we assume that progression of glomerulosclerosis over the simulation period ( 6 months or less) is minimal. Thus, $\Delta$ Perm is treated as a parameter representing damage that has already accrued but does not change during the simulation.

Glomerular hypertension also damages podocytes, causing them to leak protein. Reversible glomerular hypertensive injury to podocytes is modeled as a sigmoidal function:

$$
\mu_{g p, s e i v}=\frac{E_{\text {max }} G P_{\text {injury }}^{\gamma}}{G P_{\text {injury }}^{\gamma}-K_{m, g p \text { seiv }}^{\gamma}} .
$$

There may some podocyte injury that is irreversible or that is due to nonhemodynamic mechanisms. This is represented by a parameter $\mu_{\text {other,siev }}$. The albumin sieving coefficient is then given by

$$
K_{\text {albumin }}=K_{\text {albumino }} *\left(1+\mu_{\text {gp,seiv }}+\mu_{\text {other,seiv }}\right)
$$

where $K_{\text {albumin, } O}$ is the sieving coefficient under normal conditions. Changes in albumin excretion are assumed to reflect near-instantaneous (within hours to days) changes in glomerular hypertension. This is consistent with the fast changes in proteinuria observed with antihypertensive treatments and in diseases such as preeclampsia (Mikami et al., 2014).

Regulatory Mechanisms. The model incorporates key intrinsic and neurohormonal regulatory feedback mechanisms, as illustrated in Fig. 1 (bottom right). 1) Tubuloglomerular feedback is modeled as a signal from macula densa sodium flow (Supplemental eq. A45) that signals the afferent arteriole (Supplemental eq. A1) to constrict or relax. 2) Myogenic autoregulation is modeled as a function of preafferent pressure (Supplemental eqs. A46 and A47) that signals the preafferent arterioles (Supplemental eq. A1) to constrict or relax. 3) Vasopressin is modeled as a function of plasma $\mathrm{Na}^{+}$concentration (Supplemental eq. A48) that alters collecting duct water reabsorption (Supplemental eq. A25). 4) The pressure natriuresis phenomenon is modeled as a signal from renal interstitial hydrostatic pressure (Supplemental eqs. A49 and A50) that alters $\mathrm{Na}^{+}$reabsorption rates along the nephron (Supplemental eqs. A14 and A16). 5) Whole-body blood flow autoregulation is modeled as a signal from cardiac output that modulates peripheral resistance (eq. 12; Supplemental eq. A51). 6) To describe the renin-angiotensin-aldosterone system (RAAS), renin secretion is modeled as a function of macula densa sodium flow, with a strong inhibitory feedback from angiotensin II (AngII) bound to the Angiotensin Type 1 (AT1) receptor (AT1-bound AngII) (Supplemental eqs. A52-A55). Renin generates angiotensin I, which can be converted to AngII by ACE or chymase, or degraded (Supplemental eq. A56). AngII can bind to the AT1 or Angiotensin Type 2 (AT2) receptor, or it can be degraded (Supplemental eq. A57). AT1-bound AngII signals efferent, preafferent, and afferent vasoconstriction; PT sodium retention; and aldosterone secretion (Supplemental eq. A59). Aldosterone binds to the mineralocorticoid receptor (Supplemental eq. A60) and signals sodium retention in the connecting tubule/collecting duct (Supplemental eq. A61).

SGLT2 Inhibition. As described previously, the direct effect of $10 \mathrm{mg}$ dapagliflozin once daily on SGLT2 was modeled as a constant 85.3\% inhibitory effect on the glucose reabsorption rate per unit length through SGLT2 in the S1 and S2 segments (Supplemental eq. S68, used above in Supplemental eq. S8) (Hallow et al., 2018a). After initiating treatment with SGLT2i, urinary glucose excretion (UGE) reaches a maximum within 24 hours and then settles to a stable level slightly less than peak over the next several days. This is assumed to 
be in part due to compensation as SGLT1 and 2 are upregulated, and as described previously (Hallow et al., 2018a), we assumed unreabsorbed glucose signals upregulation of SGLT, up to a maximum increase in activity of $30 \%$ (Supplemental eqs. S68-S70). Lastly, SGLT2 i is assumed to have a weak inhibitory effect on $\mathrm{Na}^{+}$reabsorption through NHE3 (Fu et al., 2014; Pessoa et al., 2014; Coady et al., 2017) (Supplemental eq. S71). We previously showed that $8 \%$ inhibition of NHE3 with SGLT2i is sufficient to explain observed electrolyte excretion responses to SGLT2i (Hallow et al., 2018a).

\section{Technical Implementation}

The model was implemented in the open-source programming software R 3.1.2, using the $R x O D E$ package (Wang et al., 2016). Prior to availability of trial results, simulation results were placed in an online repository at https://bitbucket.org/hallowkm/dapasalt/src/master/, which provides time-stamping of the results.

\section{Virtual Patient Generation}

Baseline model parameters are given in Supplemental Tables 1-6. A population of 4000 virtual patients was generated by random sampling of a subset of model parameters over the ranges listed in Table 1. Because the distributions of these parameters within the population are generally unknown, a uniform distribution was used. Parameters to be sampled were chosen based on their mechanistic role of diabetes, kidney injury, and hypertension. Diabetes was simulated by increasing average plasma glucose concentration $\mathrm{C}_{\text {glu }}$ over a range of 7.8-14 mmol/L (corresponding to HbA1c of 6.5\%-10.5\%). Existing glomerulosclerosis and nephron loss were represented by varying the initial conditions for pressure-induced reductions in glomerular permeability $(\Delta$ Perm) and for nephron loss ( $\Delta$ nephrons), respectively. Here, $0 \%$ represents no injury, and $100 \%$ represents complete loss of glomerular permeability or nephrons, respectively. Podocyte injury $\left(\mu_{\text {other,seiv }}\right)$ and $\mathrm{PT}$ albumin reabsorptive capacity $\left(\mathrm{RC}_{\text {albumin }}\right)$ were also varied. Ranges for these parameters were chosen such that the resulting proteinuria ranged from 0 to $10 \mathrm{~g} / \mathrm{day}$. Hypertension was simulated by varying preafferent and afferent arteriole resistances, PT and collecting duct fractional $\mathrm{Na}^{+}$reabsorption, and pressure natriuresis sensitivity and renal interstitial hydrostatic pressure set point, as previously described (Hallow et al., 2014; Hallow and Gebremichael, 2017a). Sodium intake was also sampled to represent normal population variability in sodium intake. Baseline renin and aldosterone secretion (renin secretion rate, normalized aldosterone secretion rate) were varied to produce variability in baseline renin and aldosterone concentrations. After simulating to a new steady state, virtual patient values for key clinical measures were compared with physiologically reasonable values, and virtual patients with values falling outside of those ranges were rejected.

\section{Model Calibration and Validation with Diabetic Nephropathy Clinical Trials}

We have previously described calibration and validation of several key model behaviors: 1) We have calibrated the model to describe observed blood pressure reduction and plasma renin changes in response to antihypertensive therapies (ACEi including enalapril, ARBs including losartan, renin inhibitors, thiazide diuretics, and calcium channel blockers) and have shown that it is able to predict the response to combinations of these drugs (Hallow et al., 2014; Hallow and Gebremichael, 2017a). 2) We have shown that the model is able to describe clinically observed changes in urinary glucose, sodium, and volume; changes in plasma sodium and creatinine; and changes in blood pressure in response to SGLT2 inhibition (Hallow et al., 2018a). 3) We previously demonstrated that the model describes progression of albuminuria, hyperfiltration, and GFR decline in murine diabetes models (Mahato et al., 2018). However, the ability of the model to describe the effects of pharmacologic intervention in patients with diabetic nephropathy has not previously been demonstrated. To this end, we simulated several key clinical trials in diabetic nephropathy [RENAAL (Brenner et al., 2001), IDNT (Lewis et al., 2001), NESTOR (Marre et al., 2004), and AVOID (Parving et al., 2008)], focusing on the short-term ( $\leq 6$ months) albuminuria and GFR changes. Over this time period, GFR changes are likely due primarily to renal hemodynamic alterations rather than changes in disease progression (Holtkamp et al., 2011). In this analysis, we did not attempt to predict renal outcomes or long-term changes in GFR.

Each study represents a different segment of the diabetic nephropathy population and/or a different treatment regimen. RENAAL and IDNT investigated ARBs losartan and irbesartan, respectively, in patients with macroalbuminuria and low eGFR. IDNT also required that patients were hypertensive at baseline. NESTOR evaluated the ACEi enalapril in patients with microalbuminuria and moderate eGFR. In these three studies, any prior ACEi or ARB treatment was discontinued before randomization. The AVOID study investigated the addition of the renin inhibitor aliskiren to background ARB (losartan) in patients with macroalbuminuria. However, baseline

TABLE 1

Parameters varied to produce virtual patients with varying degrees of diabetes, hypertension, and kidney dysfunction

\begin{tabular}{|c|c|c|c|c|c|c|}
\hline Mechanism & Parameter & Definition & Units & Range & Median & Equation \\
\hline Diabetic increase in plasma glucose & $\mathrm{C}_{\mathrm{glu}}$ & Plasma glucose concentration & $\mathrm{mM}$ & $7.8-14$ & 9.5 & 3 \\
\hline Glomerulosclerosis & $\Delta$ Perm & Reduction in glomerular permeability & $\%$ & $0-80$ & $32 \%$ & 10 \\
\hline Nephron loss & $\Delta$ Nephrons & Initial value for nephron loss & $\%$ & $0-95$ & $51 \%$ & 2 \\
\hline Variability in PT protein reabsorption & $\mathrm{RC}_{\text {albumin }}$ & $\begin{array}{c}\text { Proxima tubule capacity for protein } \\
\text { reabsorption }\end{array}$ & $\begin{array}{l}\mathrm{pg} / \mathrm{min} \text { per } \\
\text { tubule }\end{array}$ & $1.1-2.1$ & 1.68 & 6 \\
\hline Variability in aldosterone secretion & $\mathrm{Aldo}_{0}$ & Normalized aldosterone secretion rate & - & $0.5-1.5$ & 1 & A60 \\
\hline Variability in sodium intake & $\Phi_{\mathrm{Na} \text {,in }}$ & Sodium intake rate & $\mathrm{mEq} /$ day & $80-200$ & 159 & A30 \\
\hline Increased renal vascular resistance & $\mathrm{R}_{\text {preaff }}$ & Preafferent arteriole resistance & $\mathrm{mm} \mathrm{Hg}-\mathrm{min} / \mathrm{l}$ & $14-26$ & 20 & A1 \\
\hline Increased renal vascular resistance & $\mathrm{R}_{\mathrm{aff}}$ & Afferent arteriole resistance & mm Hg-min/l & $10-17$ & 13 & $\mathrm{~A} 1$ \\
\hline Increased PT sodium reabsorption & $\eta_{\mathrm{pt}}$ & $\mathrm{PT}$ fractional $\mathrm{Na}^{+}$reabsorption rate & - & $0.6-0.84$ & 0.717 & 13 \\
\hline $\begin{array}{l}\text { Increased collecting duct sodium } \\
\text { reabsorption }\end{array}$ & $\eta_{\mathrm{cd}}$ & $\begin{array}{l}\text { Collecting duct fractional } \mathrm{Na}^{+} \\
\text {reabsorption rate }\end{array}$ & - & $0.8-0.9$ & 0.85 & A14 \\
\hline $\begin{array}{l}\text { Reduced sensitivity to pressure natriuresis } \\
\text { signals }\end{array}$ & $\mathrm{S}_{\mathrm{P}-\mathrm{N}}$ & Pressure natriuresis sensitivity & - & $0-1$ & 0.5 & A50 \\
\hline
\end{tabular}


albuminuria was less severe than in RENAAL and IDNT, and baseline eGFR fell between that of RENAAL/IDNT and NESTOR.

RENAAL was used as a calibration study, and model parameters previously calibrated using mouse data (specifically, parameters in eq. 11 defining the relationship between glomerular hydrostatic pressure and protein sieving injury) were refined to improve the fit to the RENAAL UACR data. No other model parameters required adjustment. Then, IDNT, NESTOR, and AVOID were simulated, and results were compared with reported changes in albuminuria and eGFR. It should be noted that although the model calculates GFR directly (eqs. 1 and 2), these studies estimated GFR based on serum creatinine. Equations for estimating GFR are most accurate for GFR less than $60 \mathrm{ml} / \mathrm{min}$.

\section{Clinical Trial Simulation}

For each simulated study, a subset of virtual patients was selected from the full population of virtual patients based on the trial's inclusion and exclusion criteria for $\mathrm{HbA} 1 \mathrm{c} / \mathrm{blood}$ glucose, UAER or UACR, GFR, and MAP. If more than $70 \%$ of patients in the trial were on a background antihypertensive therapy, a run-in period with that therapy was simulated before selecting the trial virtual patients. Controlled sodium intake during a run-in period was modeled when specified in the trial protocol. Treatment with study drug and dose was simulated for the trial duration or for 12 months, whichever was shortest.

\section{Summary of Calibration/Validation Studies}

RENAAL was a randomized, double-blind, placebo-controlled study of losartan in patients with type 2 diabetes (T2D) and nephropathy (UACR $>300 \mathrm{mg} / \mathrm{g}$, serum creatinine $1.3-3 \mathrm{mg} / \mathrm{dl}$ ) (Brenner et al., 2001; de Zeeuw et al., 2004). If patients were taking ACEi or ARBs at screening, these medications were discontinued and replaced by alternative antihypertensive medications (primarily diuretics and calcium channel blockers). In total, 1513 patients were randomized to $50 \mathrm{mg}$ of losartan or placebo once daily and uptitrated to $100 \mathrm{mg}$ after 4 weeks if blood pressure remained above target levels. The mean follow-up time was 3.4 years. Only changes in UACR and eGFR at 12 months were used in the current analysis.

IDNT was a randomized, double-blind, placebo-controlled study of irbesartan in patients with $\mathrm{T} 2 \mathrm{D}$, hypertension (systolic blood pressure $>135 \mathrm{~mm} \mathrm{Hg}$, diastolic blood pressure $>85 \mathrm{~mm} \mathrm{Hg}$, or documented treatment with antihypertensive), proteinuria (protein excretion $>900 \mathrm{mg} / 24$ hours), and serum creatinine $1-3 \mathrm{mg} / \mathrm{dl}$ in women and 1.2-3 mg/dl in men (Lewis et al., 2001). All ACEi, ARBs, and CCBs (calcium channel blockers) were discontinued for at least 10 days before screening and replaced with other agents. In total, 1715 patients were randomized to irbesartan titrated from 2.5 to $10 \mathrm{mg}$ per day or to placebo. The mean follow-up time was 2.6 years. Only changes in UACR and eGFR at 12 months were used in the current analysis.

NESTOR was a 1-year randomized, double-blind, placebocontrolled study of enalapril or the diuretic indapamine slow release in patients with T2D, microalbuminuria (UAER $28.8-288 \mathrm{mg} / \mathrm{day}$ ), and hypertension (systolic blood pressure 140-180 $\mathrm{mm} \mathrm{Hg}$ and diastolic blood pressure $<110 \mathrm{~mm} \mathrm{Hg}$ ) (Marre et al., 2004). All ACEi, ARBs, and CCBs (calcium channel blockers) were discontinued before randomization. In total, 570 patients were randomized to one of three groups: enalapril $10 \mathrm{mg}$, indapamine $1.5 \mathrm{mg}$ sustained release, or placebo.

AVOID was a 6-month randomized, double-blind, placebo-controlled study of aliskiren added to $100 \mathrm{mg}$ of losartan in patients with T2D and macroalbuminuria (UACR $>300 \mathrm{mg} / \mathrm{g}$ ) (Lewis et al., 2001). Inclusion criteria included eGFR $>30 \mathrm{ml} / \mathrm{min}$ per $1.73 \mathrm{~m}^{2}$ and serum creatinine $1-3 \mathrm{mg} / \mathrm{dl}$ in women and $1.2-3 \mathrm{mg} / \mathrm{dl}$ in men. During a $3-$ month run-in period, all RAAS blockers were discontinued and replaced with $100 \mathrm{mg}$ losartan daily, plus additional antihypertensives as needed to achieve target blood pressure of $<130 / 80 \mathrm{~mm} \mathrm{Hg}$.
In total, 599 patients were randomized to aliskiren (150 mg uptitrated to $300 \mathrm{mg}$ at 12 weeks) or to placebo.

DAPASALT Study Protocol. DAPASALT is an open label, mechanistic, three-arm study to evaluate the natriuretic effect of 2 weeks of dapagliflozin treatment with participants on a fixed sodium diet. The study population consists of three groups of patients (Caucasians, age 18-75 years of age) with either 1) T2D without renal impairment (HbA1c 6.5\%-10\%, eGFR 90-130 ml/min per $1.73 \mathrm{~m}^{2}$ ), 2) T2D with impaired renal function (HbA1c 6.5\%-10\%, eGFR $25-50 \mathrm{ml} /$ min per $1.73 \mathrm{~m}^{2}$ ), or 3) normoglycemic individuals with impaired renal function (eGFR $25-50 \mathrm{ml} / \mathrm{min}$ per $1.73 \mathrm{~m}^{2}$ ) and confirmed diagnosis of focal segmental glomerular sclerosis, IgA, or membranous glomerular nephropathy. For inclusion, patients must also have been treated with an ARB for at least 6 weeks prior to starting the trial, and for the individuals with T2D, a stable dose(s) of appropriate glucose-lowering medications other than SGLT2i must be present. Patients must also have stable urinary sodium excretion on two successive 24-hour measures during the run-in period. Patients with systolic and diastolic blood pressure above 160/110 mm Hg, respectively, were excluded. Full inclusion and exclusion criteria are included in the Supplemental Material. The study aims to enroll 51 patients, 17 per arm, to ensure that 15 patients complete each arm. A 2-week screening and run-in period precedes the active treatment period, and patients receive standardized meals with a sodium content of $150 \mathrm{mmol} /$ day starting at 6 days before treatment and continuing through the study. Subjects receive $10 \mathrm{mg}$ pf dapagliflozin daily for 14 days, followed by a 4-day washout period. The washout period was not considered in the analysis presented here. Study endpoints are given in Table 2. Plasma volume will be measured by indocyanine green distribution, and extracellular fluid volume will be measured by bioimpedance spectroscopy analysis.

\section{Results}

Virtual Patient Population. Of the 4000 potential virtual patients generated, 3389 had physiologically reasonable steady-state values (MAP 70-160 mm Hg, GFR 15-150 ml/ $\mathrm{min}$, UAER $0-10,000 \mathrm{mg} /$ day) and were considered acceptable. As shown in Fig. 2, top row, the distributions of baseline GFR, MAP, and UACR in the acceptable virtual patient population covered a wide range, providing a sufficient population from which to sample clinical trial populations. UAER was lognormally distributed, and GFR and MAP were normally distributed. Table 3 summarizes the number of microalbuminuric, macroalbuminuric, and hypertensive virtual patients within each GFR category. Figure 3 illustrated the effect of virtual patient differences in nephron number, glomerulosclerosis, and blood glucose on baseline GFR and UAER. As expected, GFR was lower and SNGFR was higher in virtual patients with greater nephron loss ). Virtual patients with higher glomerulosclerosis tended to have lower GFR, although some virtual patients had low GFR with minimal glomerulosclerosis (). Blood glucose was not associated with GFR (). UAER increased with moderate nephron loss, but it decreased again as nephron loss increased further (). UAER tended to be higher in virtual patients with more glomerulosclerosis (), and there was no association between blood glucose and UAER ().

To replicate trials in which patients were on an ARB therapy at baseline (AVOID and DAPASALT), virtual patients were simulated on an ARB to reach a new baseline. As shown in Fig. 2, bottom row, this shifted the virtual population distributions of UAER, GFR, and MAP to the left, but the full range of each variable was still covered. 
TABLE 2

Primary and secondary endpoints of the DAPASALT study

\begin{tabular}{|c|c|c|c|}
\hline Variable & Type & Measure & Time Points \\
\hline 24-h sodium excretion & $\begin{array}{l}\text { Primary } \\
\text { Secondary } \\
\text { Secondary }\end{array}$ & Change in mean & $\begin{array}{l}\text { Baseline vs. mean of days } 2-4 \\
\text { Baseline vs. mean of days } 12-14 \\
\text { Days } 12-14 \text { vs days } 15-17\end{array}$ \\
\hline $\begin{array}{l}\text { 24-h sodium excretion } \\
\text { 24-h sodium excretion } \\
\text { 24-h systolic blood pressure } \\
\text { 24-h systolic blood pressure } \\
\text { 24-h systolic blood pressure }\end{array}$ & Secondary & Change in mean & $\begin{array}{c}\text { Baseline vs. day } 4 \\
\text { Baseline vs. day } 13 \\
\text { Day } 13 \text { vs. day } 17\end{array}$ \\
\hline Plasma volume & Secondary & Change in mean & $\begin{array}{l}\text { Baseline vs. Day } 4 \\
\text { Baseline vs. Day } 14 \\
\text { Day } 14 \text { vs. Day } 17\end{array}$ \\
\hline Extracellular fluid volume & Secondary & Change in mean & Baseline vs. Day 14 \\
\hline
\end{tabular}

Calibration and Validation: Simulation of Previous Diabetic Nephropathy Clinical Trials. After selecting study populations from the larger virtual population by applying each trial's inclusion/exclusion criteria for albuminuria and eGFR, the virtual study populations produced were reasonably representative of the clinically reported baseline albuminuria and eGFR measures in each study (Fig. 4, top row). There was heterogeneity across studies in albuminuria measurement used (UACR or UAER) and statistic reported (geometric mean or median, S.D., interquartile range, or 95\% confidence interval), and we did not explicitly try to fit these values.

The simulated response for each trial also reproduced the reported reductions in albuminuria and eGFR. For RENAAL, model parameters were optimized to fit the observed albuminuria response. For the remaining studies, the model-predicted response reasonably reproduced the observed changes in albuminuria and eGFR. One exception to this was the AVOID
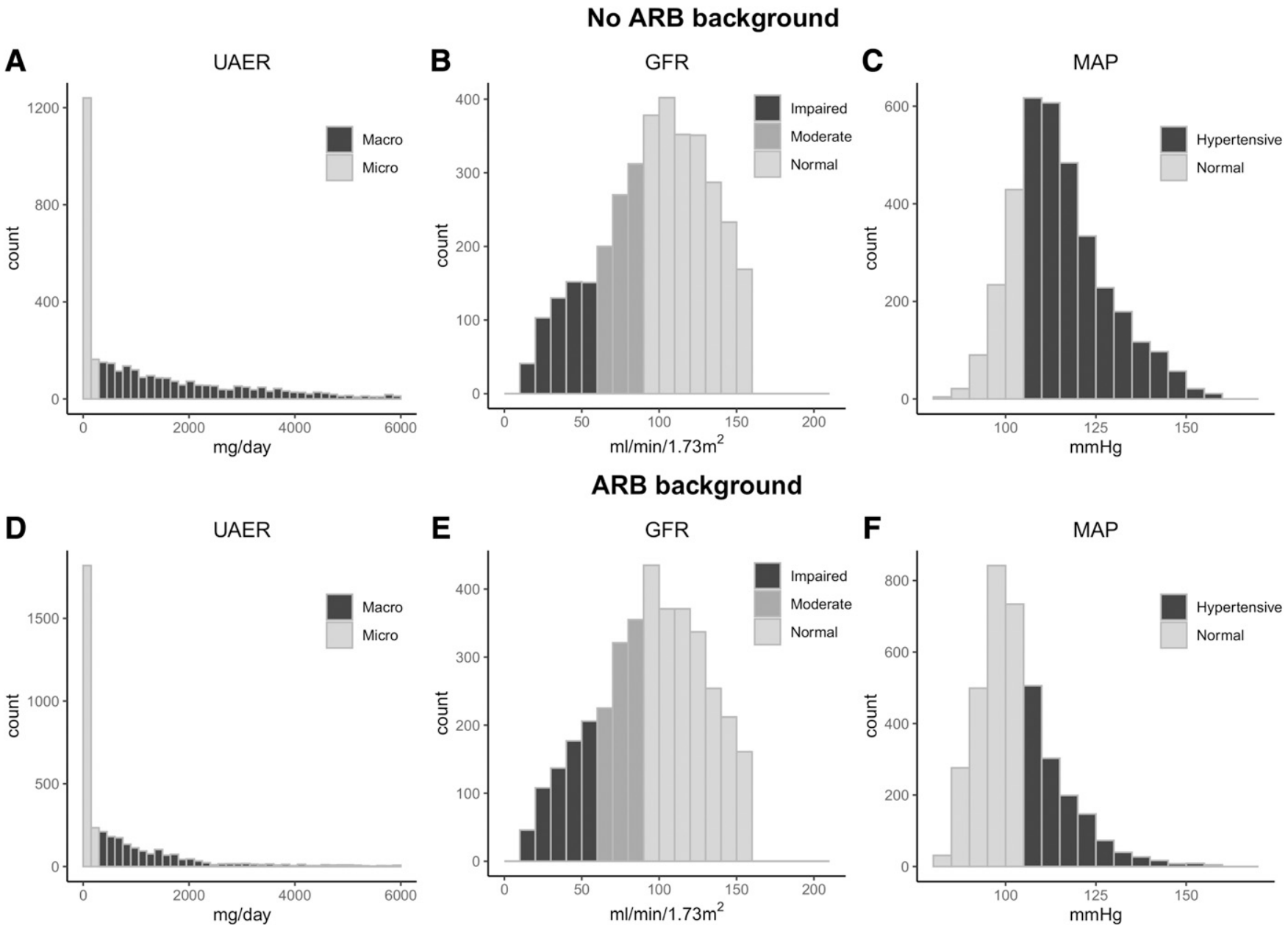

Fig. 2. (A-C) Virtual patient population covers the physiologic range of baseline UAER, GFR, and MAP. (D - F) After run-in on an ARB (losartan, 100 mg), baseline distributions are leftward-shifted but still cover a wide range. 
TABLE 3

Prevalence of albuminuria and hypertension in virtual patient population by renal function status

\begin{tabular}{|c|c|c|c|}
\hline Renal Function & Microalbuminuria & Macroalbuminuria & Hypertensive \\
\hline Impaired, GFR $<60 \mathrm{ml} / \min (n=592)$ & $43.4 \%$ & $58.0 \%$ & $83 \%$ \\
\hline Moderate impairment, GFR $60-90(n=794)$ & $53.4 \%$ & $46.5 \%$ & $71.6 \%$ \\
\hline Normal, GFR $>90 \mathrm{ml} / \mathrm{min}(n=2003)$ & $61.1 \%$ & $38.9 \%$ & $68.6 \%$ \\
\hline
\end{tabular}

GFR response. This study showed a placebo-adjusted increase in eGFR-a finding that is inconsistent with a considerable body of studies showing reductions in eGFR with RAAS blockade, both alone and in combination (Mann et al., 2008; Holtkamp et al., 2011). However, it should be noted that the model does not reproduce this unexpected behavior.

Prospective Simulation of DAPASALT. Figure 5 shows the virtual study populations for each arm in DAPASALT. The arms for T2D with preserved renal function, T2D with impaired renal function, and normoglycemic with impaired renal function will be referred to here as D-PRF, D-IRF, and N-IRF. There were no inclusion/exclusion criteria for UAER. Although each arm of the DAPASALT study will include 15-17 subjects, a larger number of virtual patients were included in the virtual population to allow the model to capture the full range of responses that might be observed.

Figures 6-8 show the simulated time course of key endpoints measured in the study for each of the three study arms, and Fig. 9 compares the response between the three groups at key time points. The washout period was not simulated. As expected, predicted 24-hour UGE is highest in the D-PRF group, lower in D-IRF, and lowest in N-IRF (median 94.6, 35.9, and $19.7 \mathrm{~g} /$ day on day 14, respectively). In all groups, 24-hour $\mathrm{Na}^{+}$excretion is predicted to peak on day 1 , overcompensate and dip just below baseline on day 2 , and then quickly return to baseline as the virtual patients again reached $\mathrm{Na}^{+}$balance. Water excretion is also predicted to peak on day 1 , but it subsequently normalizes more slowly than $\mathrm{Na}^{+}$excretion. In addition, water excretion is predicted to take longer to return to baseline in renally impaired groups (around day 14) compared with the normal renal function group (around day 7).

Urinary $\mathrm{Na}^{+}$and water excretion are not in parallel because urinary $\mathrm{Na}^{+}$excretion reflects changes in proximal tubule $\mathrm{Na}^{+}$ reabsorption, whereas water excretion reflects changes in both proximal tubule and the distal nephron. Compensatory mechanisms eventually restore both $\mathrm{Na}^{+}$and water balance, but mechanisms regulating $\mathrm{Na}^{+}$balance (e.g., renin, pressure natriuresis) achieve balance more quickly than mechanisms regulating water balance (mainly vasopressin).
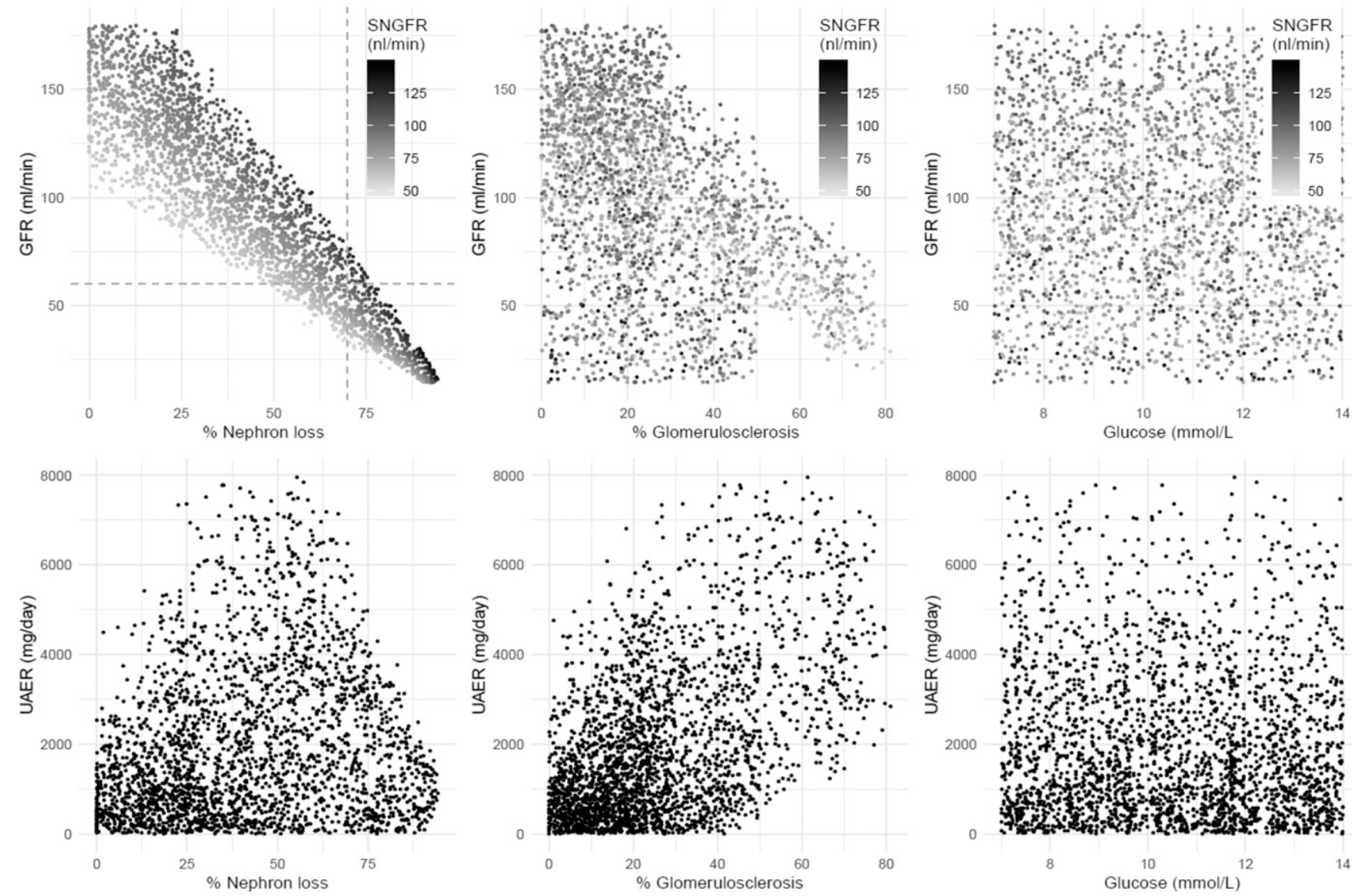

Fig. 3. Effect of virtual patient parameter values on baseline GFR and UAER. 

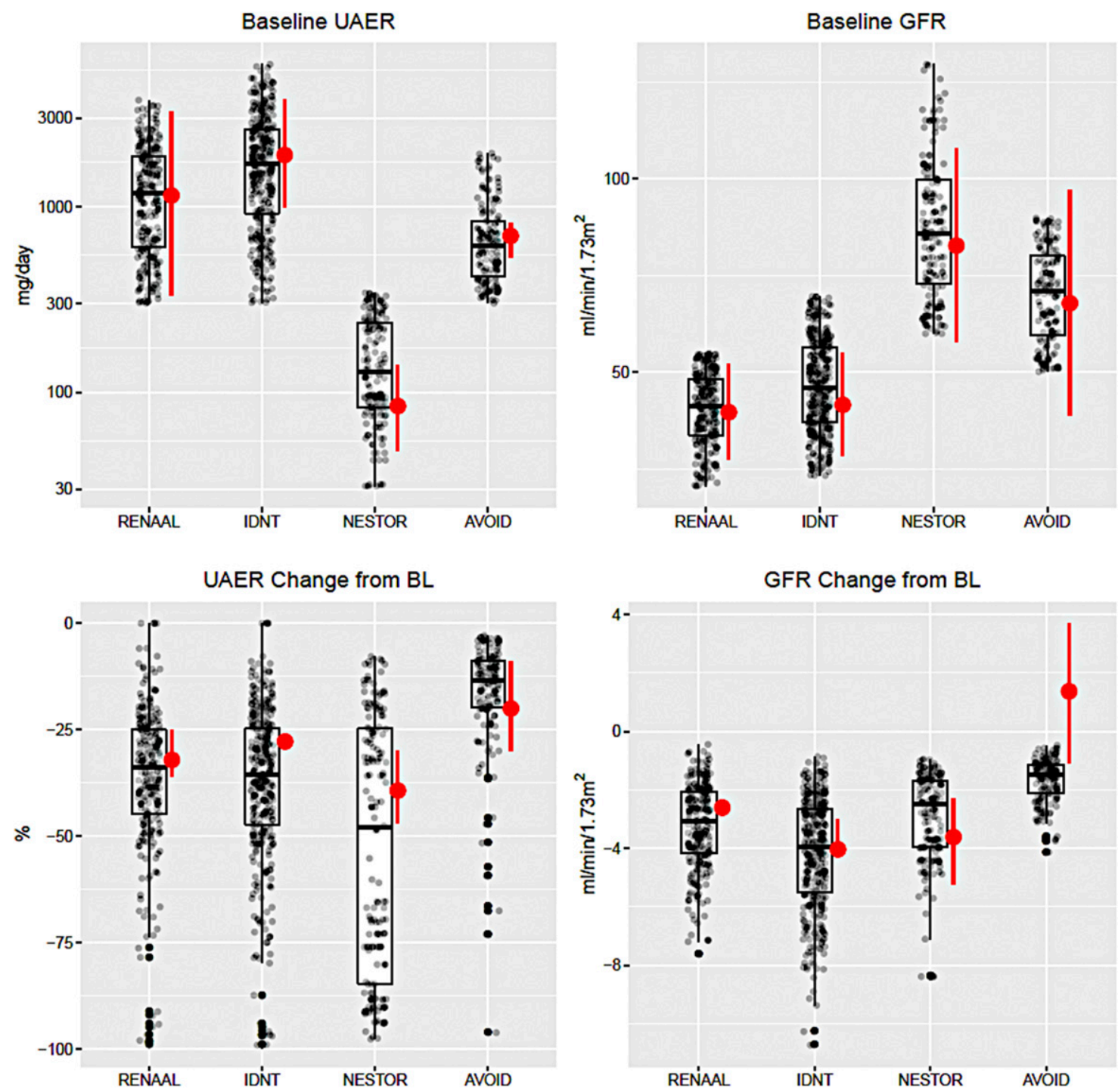

Fig. 4. Simulated and observed baseline (top row) and change from baseline (bottom row) in GFR/eGFR and UAER of key diabetic nephropathy clinical trials. Boxes: simulated median and interquartiles; gray circles: individual virtual patients; red: clinical study reported values. (RENAAL (Brenner et al., 2001): UACR is geometric mean \pm S.D.; IDNT (Lewis et al., 2001): UAER median and interquartile range, no measure of variability reported for change in UAER; NESTOR (Marre et al., 2004): UAER geometric mean and interquartile range; AVOID (Parving et al., 2008): UAER geometric mean and $95 \%$ confidence interval. For all studies, eGFR is mean \pm S.D.. No S.D. reported for RENAAL change in eGFR.)

The decrease in MAP in D-PRF is predicted to be slightly larger than in the D-IRF group ( -5.1 vs. $-3.6 \mathrm{~mm} \mathrm{Hg}$ ). MAP reduction in the $\mathrm{N}$-IRF group is predicted to be small $(1 \mathrm{~mm} \mathrm{Hg})$.

Our simulations predict that the initial reduction in GFR will be much smaller in the impaired renal function groups $(-3.8$ and $-2.3 \mathrm{ml} / \mathrm{min}$ in D-IRF and N-IRF groups, respectively) compared with the D-PRF group $(-15.2 \mathrm{ml} / \mathrm{min})$. The initial reduction in GFR also varied widely within the D-PRF group, as indicated by the width of the interquartile range (Fig. 6). Further analysis showed that the largest drops occurred in hyperfiltering virtual patients (baseline GFR $>110 \mathrm{ml} / \mathrm{min}$, analysis not shown).

UAER is predicted to decrease substantially in all three groups. In patients with diabetes, the UAER reduction is predicted to be less but still quite large in the impaired renal function group (34.8\%) compared with the normal renal function group (45.8\%). A smaller reduction (14.2\%) is predicted in the N-IRF group. Our simulations predict that the maximum UAER reduction will occur within 14 days.
As we have modeled previously, reductions in IFV are predicted to be much greater than reductions in blood volume. Predicted blood volume reduction is largest in the D-PRF $(210 \mathrm{ml})$, smaller in D-IRF (150 ml), and smallest in the N-IRF group $(40 \mathrm{ml})$. On the other hand, predicted IFV reduction is larger in the D-IRF group than in the D-PRF group (1.81 vs. 1.681) and was still substantially reduced in the N-IRF group (11). Thus, the ratio of IFV to blood volume reduction is predicted to be larger in the renal impairment groups than in normal renal function.

\section{Discussion}

Clinical Implications of Model Predictions. Given the weaker glycosuria response to SGLT2i in patients with renal impairment and in nonpatients with diabetes, volume changes resulting from osmotic diuresis with SGLT2i might be expected to be diminished in these populations. However, the model predicts IFV reduction will be similar in T2D with 

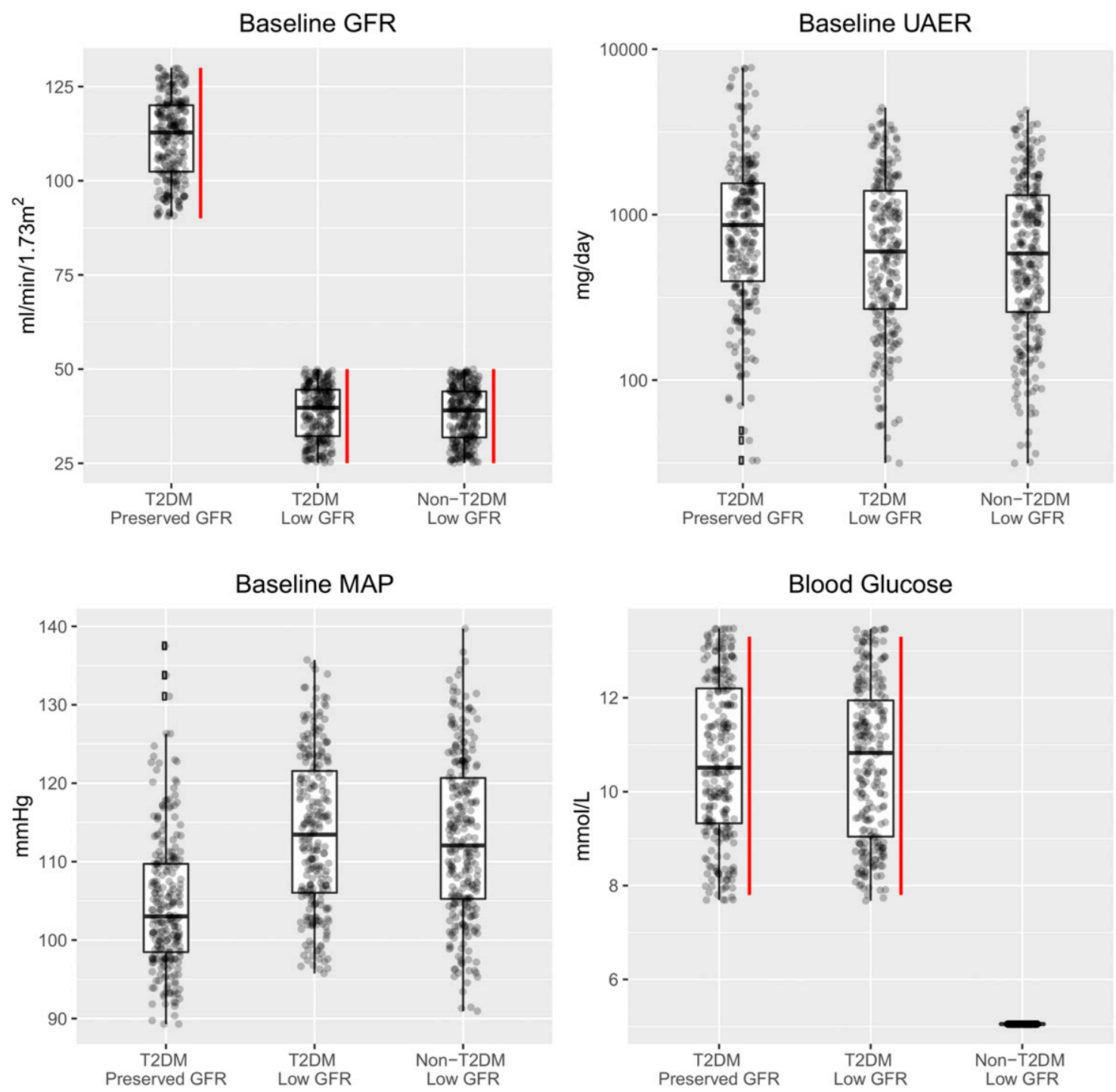

Fig. 5. Baseline characteristics of DAPASALT virtual study arms (T2D preserved GFR $n=250$, T2D low GFR $n=250$, non-T2D low GFR $n=272$ ). Red bars: study inclusion exclusion criteria.

and without renal impairment and that nondiabetics with renal impairment will see smaller but still substantial IFV reductions, even with much lower UGE. Assuming IFV plays an important role in the cardiovascular benefits of SGLT2i, this is consistent with recent findings of the DAPA-HF (Dapagliflozin in Patients with Heart Failure and Reduced Ejection Fraction) study, in which significant improvements in the primary endpoint (worsening of heart failure or cardiovascular death) were seen across all baseline GFRs and independent of diabetic status (McMurray et al., 2019).

The model also suggests a mechanistic explanation for these predictions. Within a single nephron, predicted changes in water excretion were similar between D-PRF and D-IRF groups. However, because the D-PRF have more functioning nephrons, the initial peak in water excretion in this group was larger (Fig. 6). The modeling suggests this causes a larger vasopressin response, which limits further excretion and quickly returns water excretion to baseline. In D-IRF, the predicted initial water excretion and thus vasopressin response is lower, so compensation occurs more slowly, allowing similar total water excretion and thus similar volume changes as in the D-PRF group, even though the initial peak was smaller.

A second finding, which we demonstrated previously in single virtual patients (Hallow et al., 2018a), is that glomerular hydrostatic pressure reductions, which likely play a large role in dapagliflozin's renoprotective effects, are predicted to be similar in patients with normal or impaired renal function, and initial GFR drop is expected to be smaller in impaired renal function. The sustained glomerular pressure reduction likely explains why the antiproteinuric effects are sustained in patients with low GFR (Heerspink et al., 2016; Fioretto et al., 2018).

Comparison with Available Data. Although DAPASALT study results are not yet available, several available data support the predicted responses. Our simulations reproduce higher UGE observed in patients with normal versus impaired renal function (List et al., 2009; Kohan et al., 2014). Predicted MAP reductions of $3-5 \mathrm{~mm} \mathrm{Hg}$ are consistent with previous studies (List et al., 2009; Wilding et al., 2009; 

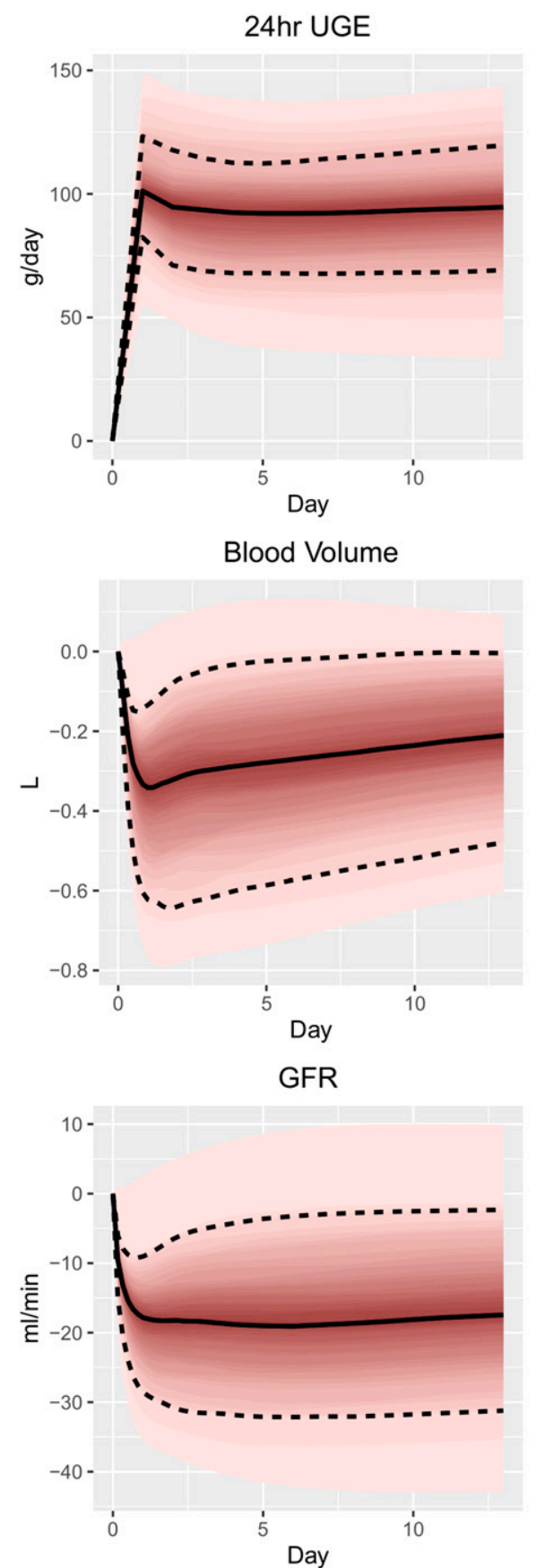

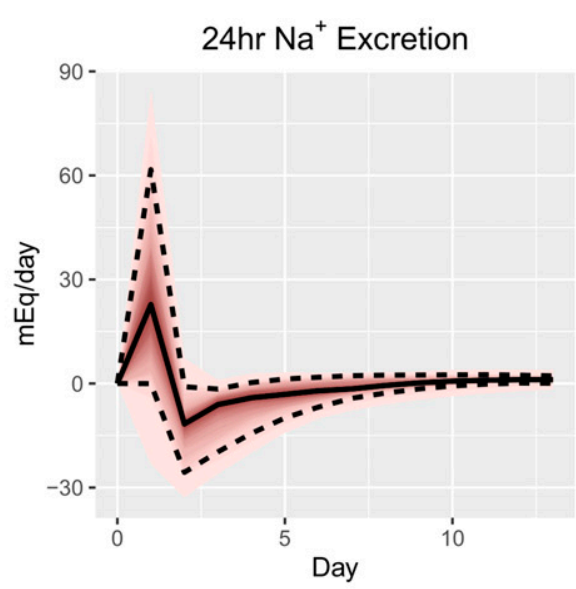

Interstitial Fluid Volume

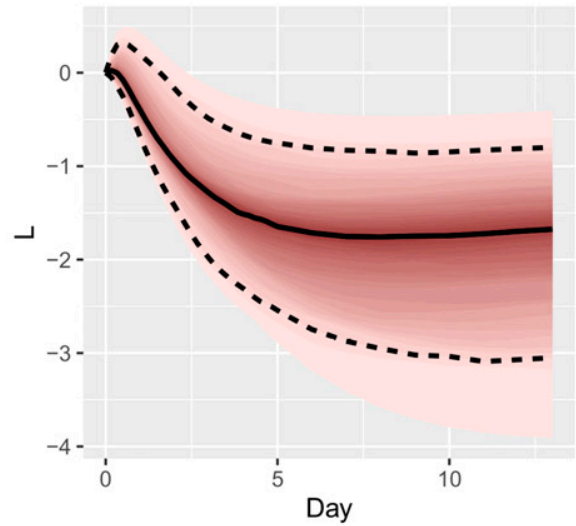

UAER

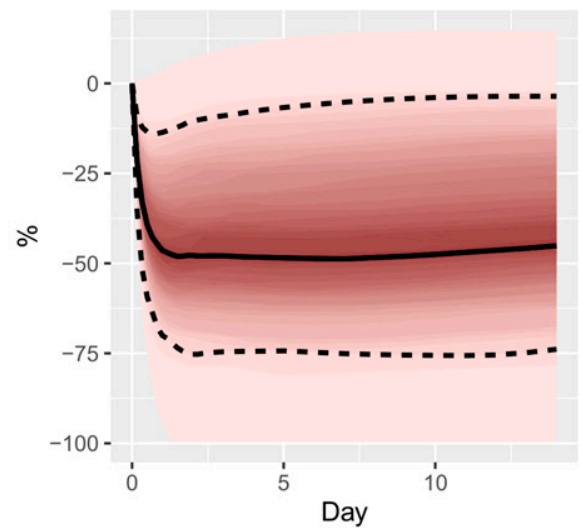

24 hr Water Excretion

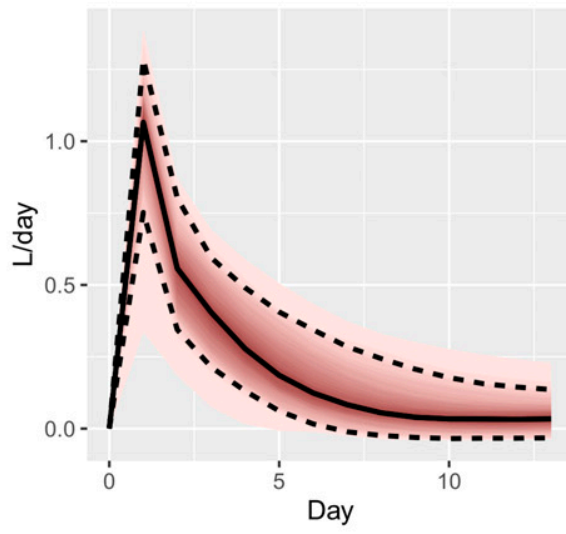

MAP

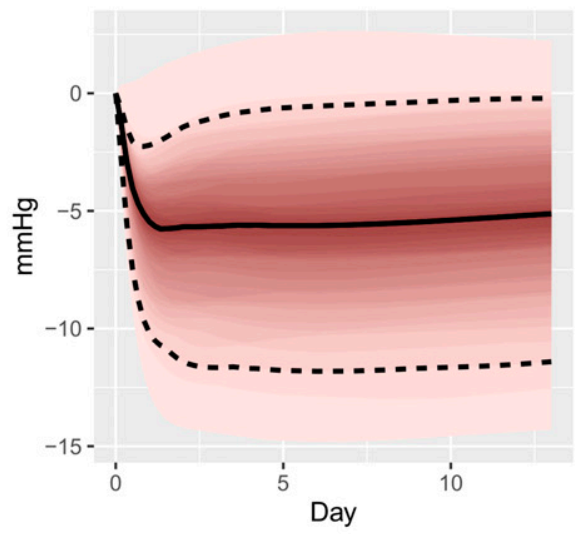

Glomerular Hydrostatic Pressure

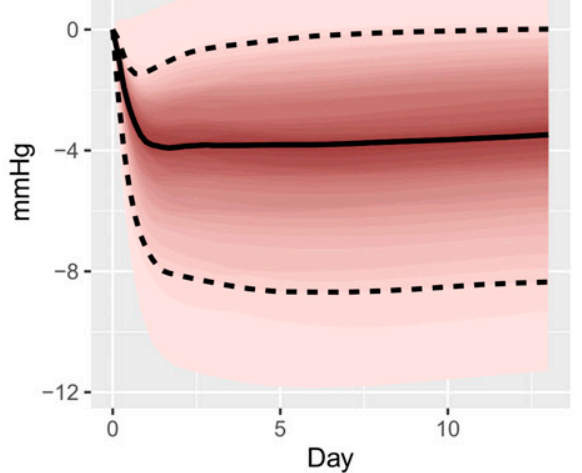

Fig. 6. DAPASALT D-PRF arm. Simulated time course for change from baseline with $10 \mathrm{mg}$ dapagliflozin. Solid line: median; dashed lines: $25 \%-75 \%$; pink bands: $0 \%-100 \%$ range of response.

Ferrannini et al., 2010). The simulations reproduce the well known initial drop in GFR with SGLT2i initiation. This reversible initial reduction is followed by a much slower rate of GFR decline (Wanner et al., 2016). Our simulations predict the initial GFR drop with dapagliflozin will be smaller in D-IRF than D-PRF. The predicted magnitude in D-IRF $(-3.8 \mathrm{ml} / \mathrm{min})$ is consistent with reported eGFR changes in clinical studies of diabetic chronic kidney disease (CKD). In the DERIVE study, in patients with T2D with stage $3 \mathrm{a}$ CKD treated with dapagliflozin, GFR fell by $5 \mathrm{ml} / \mathrm{min}$ per $1.73 \mathrm{~m}^{2}$ at week 4 (Fioretto et al., 2018). A similar reduction $(-4 \mathrm{ml} / \mathrm{min}$ per $1.73 \mathrm{~m}^{2}$ ) was observed with canagliflozin at week 6 in T2D with stage $3 \mathrm{CKD}$ (Yamout et al., 2014). Another small study in patients with more severe CKD (mean eGFR $30.3 \mathrm{ml} / \mathrm{min}$ per $\left.1.73 \mathrm{~m}^{2}\right)$ found a smaller $\left(1.3 \mathrm{ml} / \mathrm{min}\right.$ per $\left.1.73 \mathrm{~m}^{2}\right)$ reduction. This is consistent with our predicted smaller initial GFR reduction in patients with lower baseline GFR.

Most studies reporting renal function changes with SGLT2i have used serum creatinine to estimate GFR, although a few used inulin clearance or other methods to measure GFR directly. eGFR is accurate for GFR less than $60 \mathrm{ml} / \mathrm{min}$ per $1.73 \mathrm{~m}^{2}$ but may be less accurate for higher GFRs. Studies reporting eGFR changes in patients without renal impairment have reported reductions of 4 to $5 \mathrm{ml} / \mathrm{min}$ per $1.73 \mathrm{~m}^{2}$ 
24hr UGE

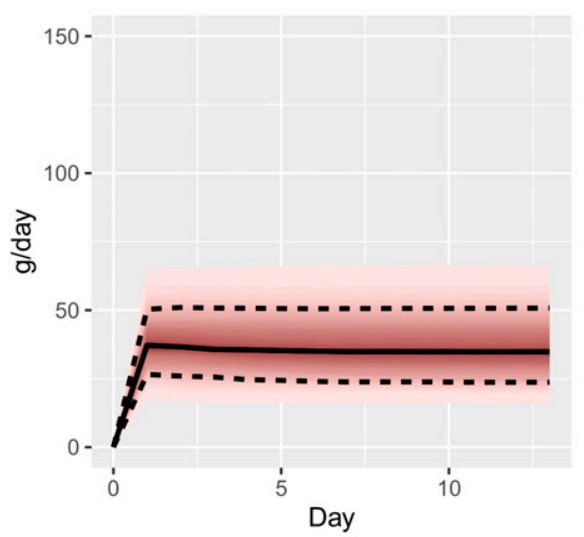

Blood Volume
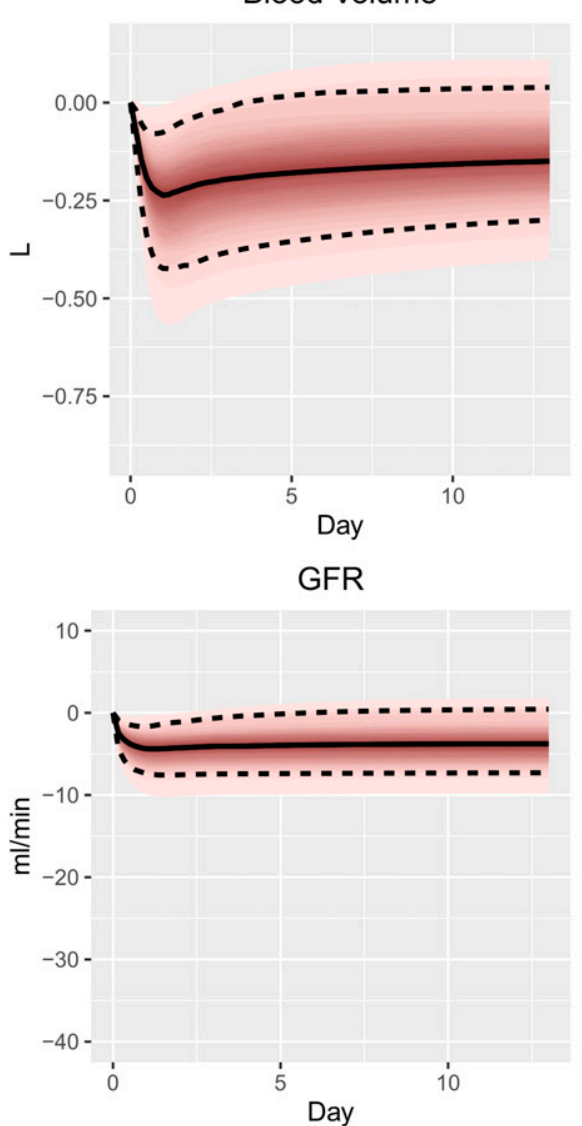
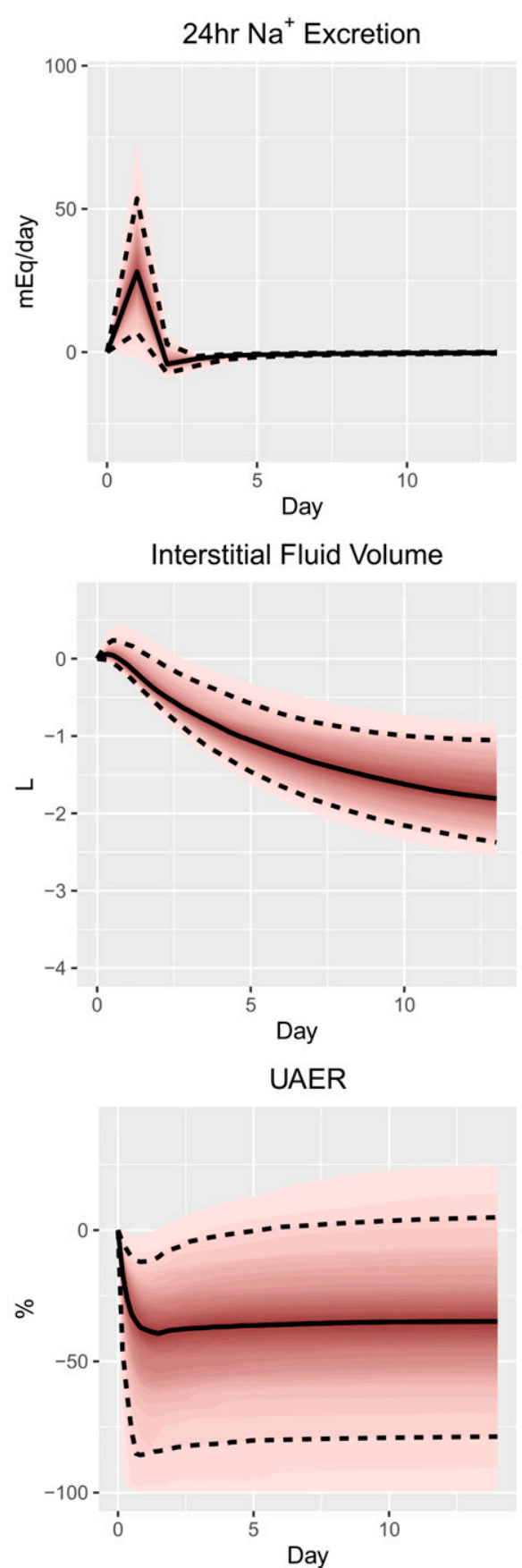

$24 \mathrm{hr}$ Water Excretion

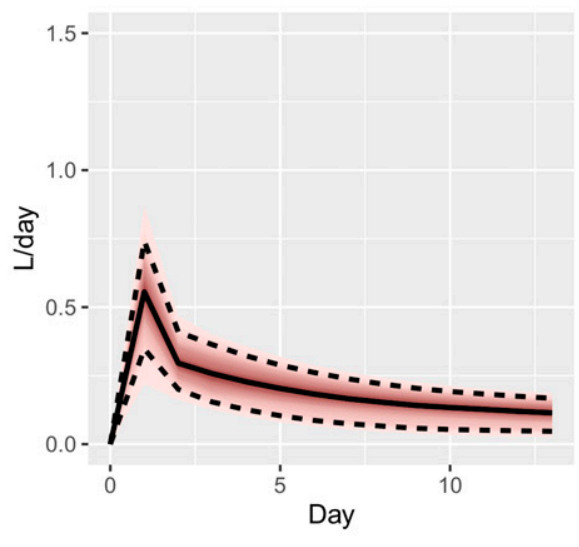

MAP

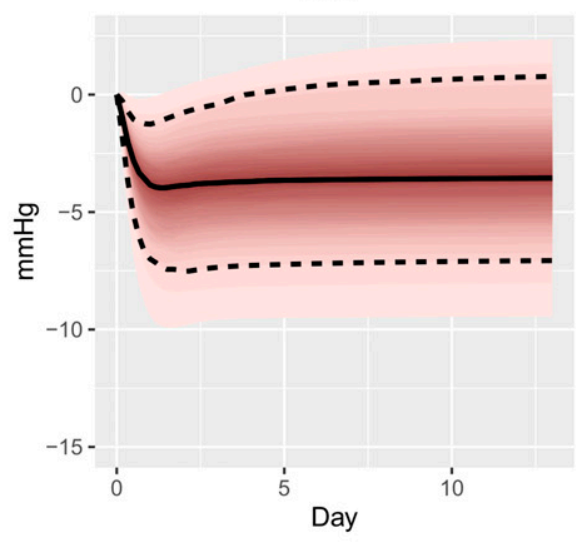

Glomerular Hydrostatic Pressure

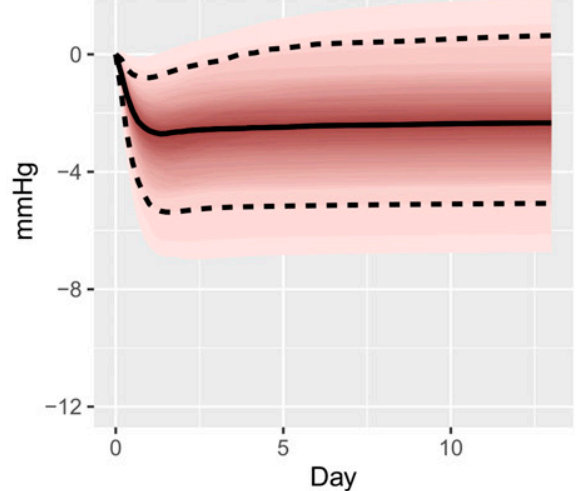

Fig. 7. DAPASALT D-IRF arm. Simulated time course for change from baseline with $10 \mathrm{mg}$ dapagliflozin. Solid line: median; dashed lines: $25 \%-75 \%$; pink bands: $0 \%-100 \%$ range of response.

(Heerspink et al., 2016), and pooled analyses have shown no dependence of change in eGFR on baseline eGFR (Petrykiv et al., 2017). However, studies that measured GFR directly have found larger reductions. In one study, GFR dropped by $10.8 \mathrm{ml} / \mathrm{min}$ initially in patients with T2D and normal renal function treated with dapagliflozin (Lambers Heerspink et al., 2013). Another study reported reductions of 5, 10, and $12 \mathrm{ml} / \mathrm{min}$ in fasted, euglycemic, and hyperglycemic states, respectively (van Bommel et al., 2020). Cherney et al. (2014) found that empagliflozin reduced GFR in hyperfiltering patients with type 1 diabetes by $25-45 \mathrm{ml} / \mathrm{min}$ per $1.73 \mathrm{~m}^{2}$, depending on glycemic state. They found no GFR change in nonhyperfiltering patients.
The magnitude of changes predicted in the DAPASALT D-PRF group $(-15.2 \mathrm{ml} / \mathrm{min})$ are consistent with studies measuring GFR directly, and it is possible that measured changes in eGFR in DAPASALT may underpredict true changes in GFR. Our simulations are also consistent with a larger initial GFR drop in hyperfiltering than nonhyperfiltering patients.

The model-predicted changes in albuminuria are consistent with studies showing consistent proteinuria reduction with SGLT2i. In patients with T2D and moderate renal function (baseline eGFR of 72-82 ml/min per $1.73 \mathrm{~m}^{2}$ ), $10 \mathrm{mg}$ dapagliflozin reduced UACR by $45 \%$ at week (Heerspink et al., 2016) and reduced 24-hour UAER by $36.2 \%$ at 6 weeks (Petrykiv 

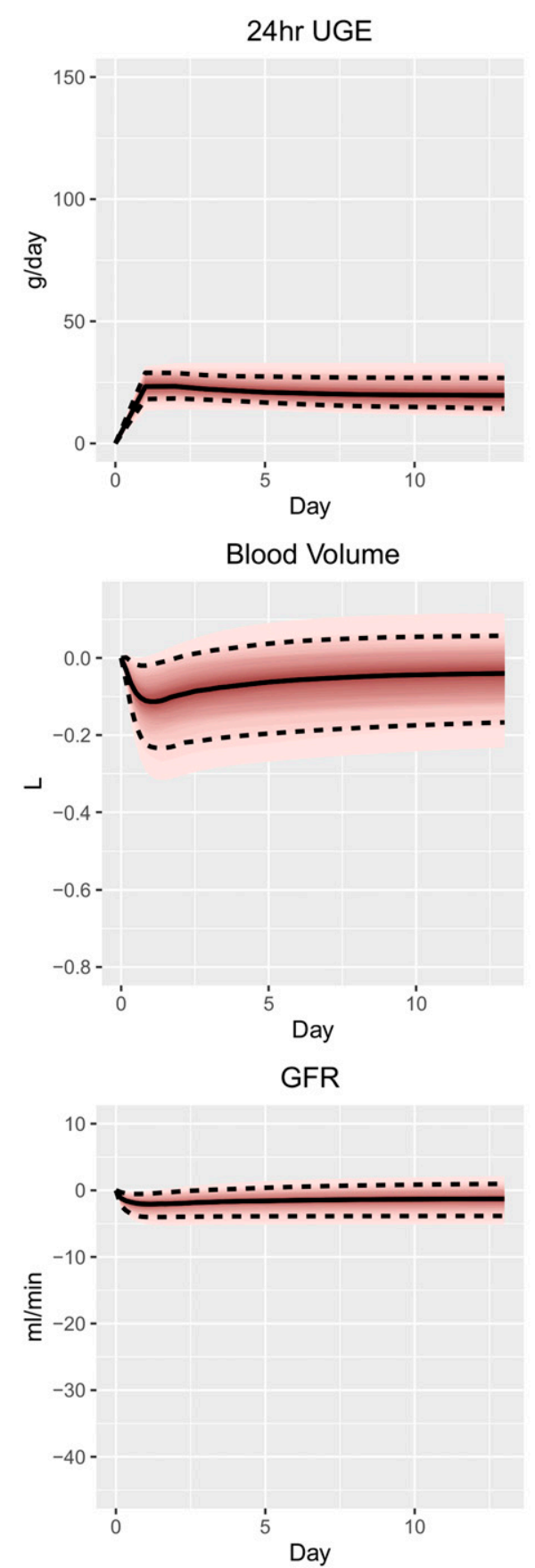

$24 \mathrm{hr} \mathrm{Na}^{+}$Excretion
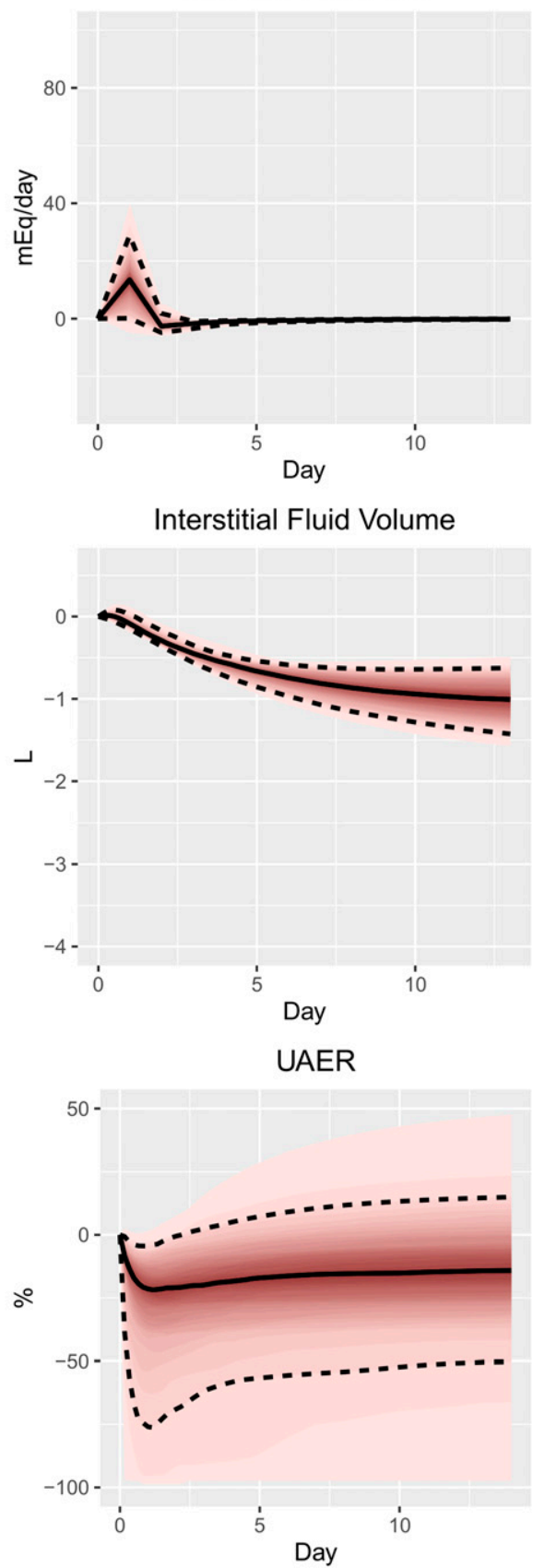

$24 \mathrm{hr}$ Water Excretion
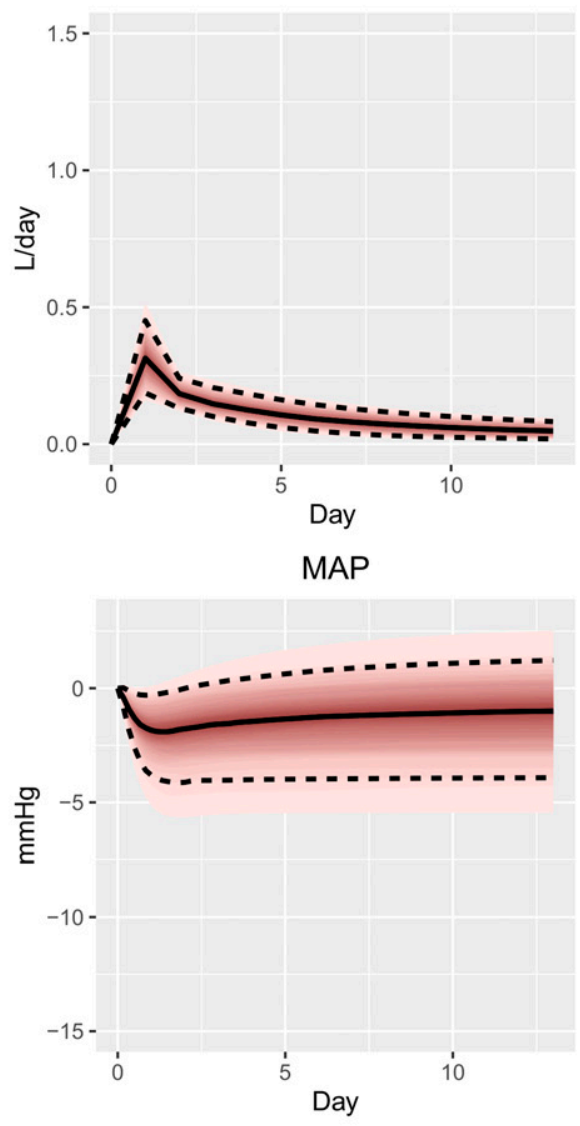

Glomerular Hydrostatic Pressure

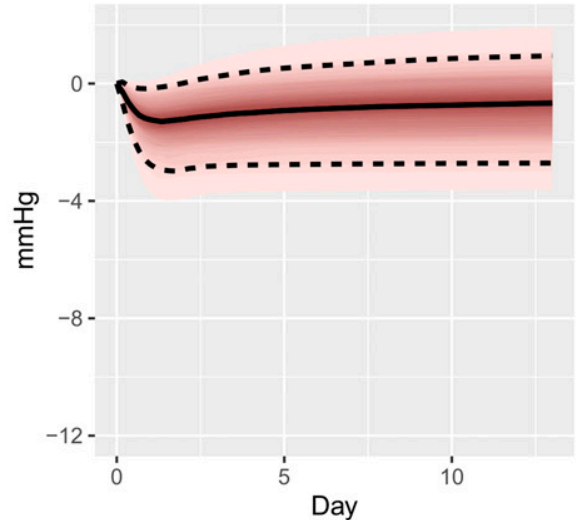

Fig. 8. DAPASALT N-IRF arm. Simulated time course for change from baseline with $10 \mathrm{mg}$ dapagliflozin. Solid line: median; dashed lines: $25 \%-75 \%$; pink bands: $0 \%-100 \%$ range of response.

et al., 2017). In T2D patients with stage $3 \mathrm{a}$ CKD and albuminuria, UACR fell $30.7 \%$ at week 4 and $41.7 \%$ by week 12 (Fioretto et al., 2018), whereas in stage 3b-4 CKD, UACR was reduced $38.4 \%$ over 102 weeks. Our predicted reductions of $45 \%$ and $35 \%$ in $\mathrm{T} 2 \mathrm{D}$ with normal and impaired renal function, respectively, are consistent with these findings.

Fewer data are available on fluid volume changes with SGLT2i. As we predict here and in previous analyses of single virtual patients (Hallow et al., 2018a,b), SGLT2i may elicit much larger relative reductions in IFV than in blood volume. This decongestive effect without excessive reduction in blood pressure and organ perfusion may explain the unexpectedly large benefits on heart failure (Zinman et al., 2015; McMurray et al., 2019). To our knowledge, DAPASALT will be the first to measure changes in both IFV and blood volume in the same study. However, studies have separately reported measures that reflect blood or total extracellular fluid volume change. SGLT2i have consistently been found to increase hematocrit, suggesting blood volume reduction. Hematocrit increases of $1.3 \%$ and $2.2 \%$ were reported in $\mathrm{T} 2 \mathrm{D}$ with normal renal function (Lambers Heerspink et al., 2013; Wada et al., 2019). If red blood cell volume remains constant, the model-predicted changes in blood volume correspond to $1.7 \%$ hematocrit increase in $\mathrm{T} 2 \mathrm{D}$ with preserved GFR, consistent with these 
$\Delta \mathrm{UGE}$ - Day 14

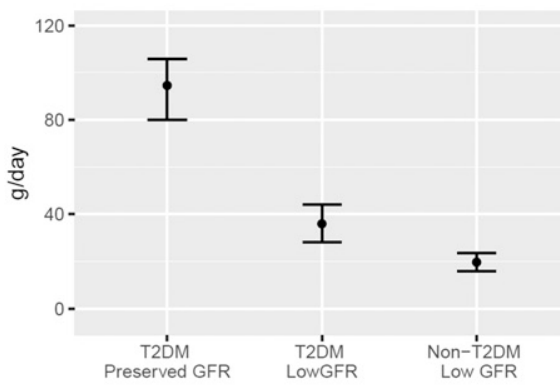

$\Delta$ Blood Volume - Day 14

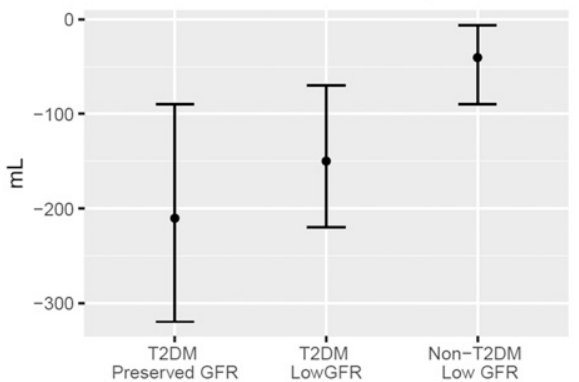

$\Delta$ GFR - Day 14

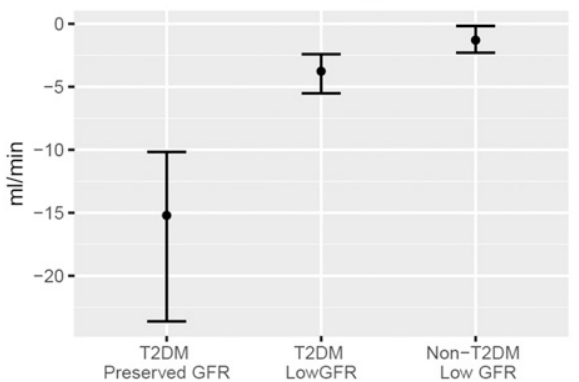

$\Delta \mathrm{Na}^{+}$Excretion

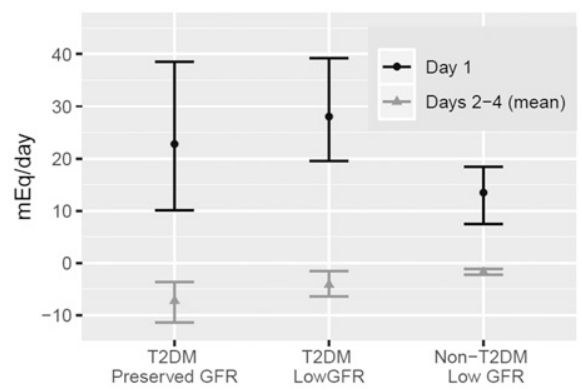

$\Delta$ Interstitial Fluid Volume - Day 14

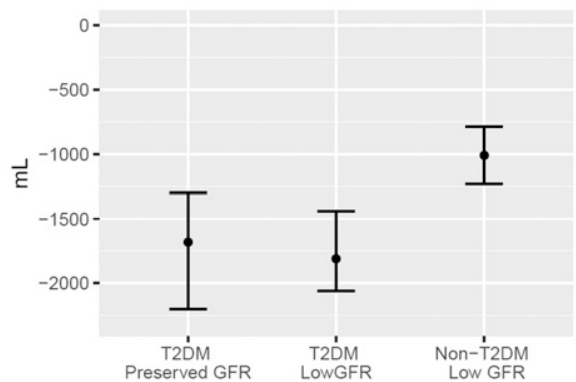

$\triangle$ UAER - Day 14

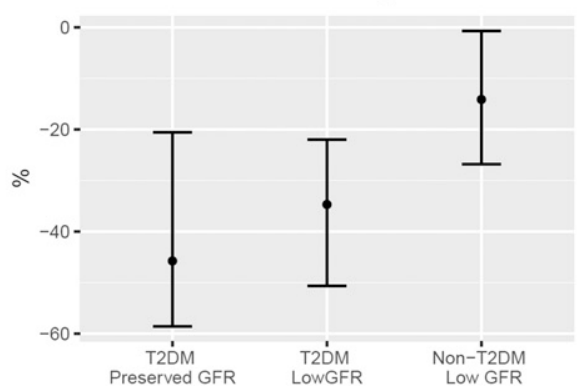

$\Delta$ Water Excretion

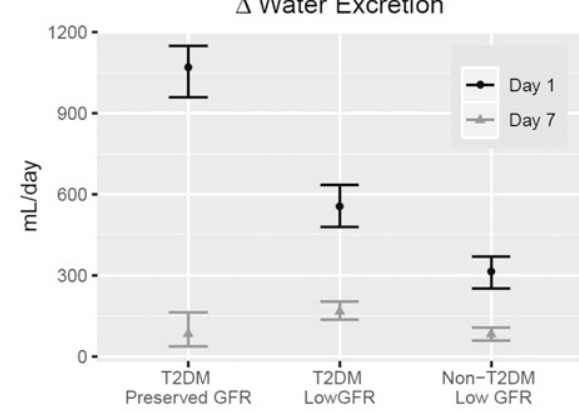

$\Delta$ Mean Arterial Pressure - Day 14

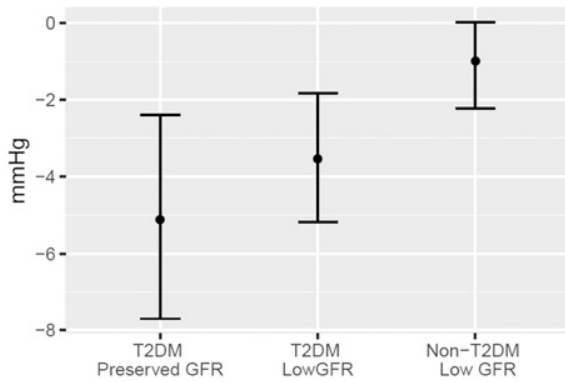

$\Delta$ Glomerular Hydrostatic Pressure - Day 14

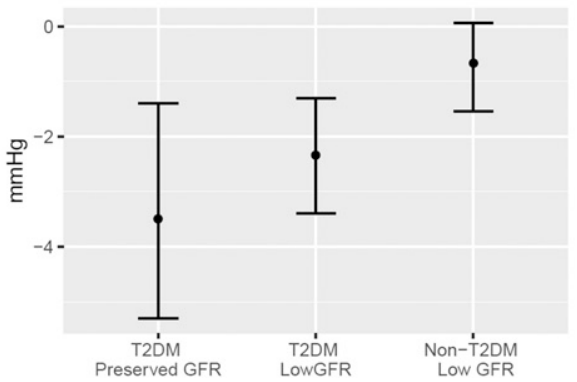

Fig. 9. Simulated response to daily dosing of $10 \mathrm{mg}$ dapagliflozin in DAPASALT study arms. All data are medians and interquartile ranges.

studies. Hematocrit changes may also reflect changes in hematopoiesis (Maruyama et al., 2019), but these effects were not modeled here. Two recent studies used bioimpedance to measure extracellular water changes. Unfortunately, these studies did not report hematocrit, so relative reductions in blood and interstitial volumes cannot be determined. These studies were nonrandomized and were not placebo-controlled and thus should be interpreted with care. In Ohara et al. (2019), extracellular water was reduced by $8.4 \%$ in patients with diabetes with impaired renal function treated with dapagliflozin, and our simulations predict a $9.5 \%$ reduction. A recent observational study in T2D with normal renal function treated with empagliflozin or dapagliflozin reported a smaller reduction $\left(400 \mathrm{ml} / 1.73 \mathrm{~m}^{2}\right)$ at day 3 . A third study with tofogliflozin showed a $0.3-\mathrm{kg}$ reduction in extracellular water (Kamei et al., 2018). This study actually showed a nonsignificant hematocrit decrease, inconsistent with other studies consistently showing increases.

Model Validation. Models cannot reproduce all aspects of physiology and disease. Making predictions and comparing with clinical data are ways to determine whether the model is "good enough" or whether important mechanisms are missing. We previously showed that the model reproduces biomarker and blood pressure responses to RAAS blockers, diuretics, and calcium channel blockers in hypertension (Hallow et al., 2014) and urinary and serum biomarker responses to dapagliflozin in normal subjects (Hallow et al., 2018a). Here, we further retrospectively validated the kidney injury and albuminuria components of the model by demonstrating reasonable agreement between model predictions and observed changes in albuminuria and eGFR for previous diabetic nephropathy clinical trials. This validation demonstrated that the renal physiology/pathophysiology/pharmacology represented in the model is sufficient for describing responses in this population and provides confidence for making prospective predictions in similar populations treated with SGLT2i.

Limitations. The model captures some but not all sources of variability in SGLT2i response. Thus, predicted interquartile ranges are likely narrower than true interquartile ranges. DAPASALT virtual patients were selected based on inclusion/ exclusion criteria. Because we do not know the true baseline characteristics, virtual and real populations may differ. In particular, because no limits were placed on UACR in the DAPASALT protocol, virtual and real baseline UACR could be quite different, which could impact predicted treatment responses. Few studies report time courses for albuminuria 
changes prior to 4 weeks. If the model overestimates the speed of UAER reduction, the 2 -week UAER response may be overpredicted. For the normoglycemic arm, we did not distinguish between mechanisms of IgA nephropathy, focal segmental glomerular sclerosis, or membranous glomerular nephropathy. Once study results are available, comparison of simulated and observed baseline characteristics and responses may provide further information for better modeling these populations.

\section{Conclusions}

The model predicts similarly large IFV reduction between D-PRF and D-IRF and less, but still substantial, IFV reduction in N-IRF, even though glycosuria is attenuated in groups with impaired renal function. When DAPASALT results become available, comparison with these prospective simulations will provide a basis for evaluating how well we understand the renal and volume homeostasis mechanism(s) of SGLT2i. If the prospective simulations predict the results well, this will also provide further validation of the model as a tool for future predictions.

\section{Authorship Contributions}

Participated in research design: Hallow, Boulton, Penland, Helmlinger, Nieves, Heerspink, Greasley.

Conducted Experiments: Hallow, Nieves.

Contributed new reagents or analytic tools: Hallow.

Performed data analysis: Hallow, Nieves.

Wrote or contributed to the writing of the manuscript: Hallow, Boulton, Penland, Helmlinger, van Raalte, Heerspink, Greasley.

\section{References}

Bivona BJ, Park S, and Harrison-Bernard LM (2011) Glomerular filtration rate determinations in conscious type II diabetic mice. Am J Physiol Renal Physiol 300: F618-F625.

Brenner BM, Cooper ME, de Zeeuw D, Keane WF, Mitch WE, Parving HH, Remuzzi G, Snapinn SM, Zhang Z, and Shahinfar S; RENAAL Study Investigators (2001) Effects of losartan on renal and cardiovascular outcomes in patients with type 2 diabetes and nephropathy. N Engl J Med 345:861-869.

Cherney DZ, Perkins BA, Soleymanlou N, Maione M, Lai V, Lee A, Fagan NM, Woerle HJ, Johansen OE, Broedl UC, et al. (2014) Renal hemodynamic effect of sodium-glucose cotransporter 2 inhibition in patients with type 1 diabetes mellitus. Circulation 129:587-597.

Coady MJ, El Tarazi A, Santer R, Bissonnette P, Sasseville LJ, Calado J, Lussier Y, Dumayne C, Bichet DG, and Lapointe JY (2017) MAP17 is a necessary activator of renal Na+/glucose cotransporter SGLT2. J Am Soc Nephrol 28:85-93.

Dekkers CCJ, Petrykiv S, Laverman GD, Cherney DZ, Gansevoort RT, and Heerspink HJL (2018) Effects of the SGLT-2 inhibitor dapagliflozin on glomerular and tubular injury markers. Diabetes Obes Metab 20:1988-1993.

de Zeeuw D, Remuzzi G, Parving HH, Keane WF, Zhang Z, Shahinfar S, Snapinn S, Cooper ME, Mitch WE, and Brenner BM (2004) Proteinuria, a target for renoprotection in patients with type 2 diabetic nephropathy: lessons from RENAAL. Kidney Int 65:2309-2320.

Ferrannini E, Ramos SJ, Salsali A, Tang W, and List JF (2010) Dapagliflozin monotherapy in type 2 diabetic patients with inadequate glycemic control by diet and exercise: a randomized, double-blind, placebo-controlled, phase 3 trial. Diabetes Care 33:2217-2224.

Fioretto P, Del Prato S, Buse JB, Goldenberg R, Giorgino F, Reyner D, Langkilde AM, Sjöström CD, and Sartipy P; DERIVE Study Investigators (2018) Efficacy and safety of dapagliflozin in patients with type 2 diabetes and moderate renal impairment (chronic kidney disease stage 3A): the DERIVE Study. Diabetes Obes Metab 20:2532-2540.

Fioretto P, Giaccari A, and Sesti G (2015) Efficacy and safety of dapagliflozin, a sodium glucose cotransporter 2 (SGLT2) inhibitor, in diabetes mellitus. Cardiovasc Diabetol 14:142.

Flyvbjerg A, Dagnaes-Hansen F, De Vriese AS, Schrijvers BF, Tilton RG, and Rasch $R$ (2002) Amelioration of long-term renal changes in obese type 2 diabetic mice by a neutralizing vascular endothelial growth factor antibody. Diabetes 51:3090-3094.

Fu Y, Gerasimova M, Mayoux E, Masuda T, and Vallon V (2014) SGLT2 inhibitor empagliflozin increases renal NHE3 phosphorylation in diabetic Akita mice: possible implications for the prevention of glomerular hyperfiltration (Abstract). $\mathrm{Di}$ abetes 63:132.

Guthrie R (2018) Canagliflozin and cardiovascular and renal events in type 2 diabetes. Postgrad Med 130:149-153.
Hallow KM and Gebremichael Y (2017a) A quantitative systems physiology model of renal function and blood pressure regulation: application in salt-sensitive hypertension. CPT Pharmacometrics Syst Pharmacol 6:393-400.

Hallow KM and Gebremichael Y (2017b) A quantitative systems physiology model of renal function and blood pressure regulation: model description. CPT Pharmacometrics Syst Pharmacol 6:383-392.

Hallow KM, Gebremichael Y, Helmlinger G, and Vallon V (2017) Primary proximal tubule hyperreabsorption and impaired tubular transport counterregulation determine glomerular hyperfiltration in diabetes: a modeling analysis. Am J Physiol Renal Physiol 312:F819-F835.

Hallow KM, Greasley PJ, Helmlinger G, Chu L, Heerspink HJL, and Boulton DW (2018a) Evaluation of renal and cardiovascular protection mechanisms of SGLT2 inhibitors: model-based analysis of clinical data. Am J Physiol Renal Physiol 315: F1295-F1306

Hallow KM, Helmlinger G, Greasley PJ, McMurray JJV, and Boulton DW (2018b) Why do SGLT2 inhibitors reduce heart failure hospitalization? A differential volume regulation hypothesis. Diabetes Obes Metab 20:479-487.

Hallow KM, Lo A, Beh J, Rodrigo M, Ermakov S, Friedman S, de Leon H, Sarkar A Xiong Y, Sarangapani R, et al. (2014) A model-based approach to investigating the pathophysiological mechanisms of hypertension and response to antihypertensive therapies: extending the Guyton model. Am J Physiol Regul Integr Comp Physiol 306:R647-R662.

Hammon M, Grossmann S, Linz P, Kopp C, Dahlmann A, Garlichs C, Janka R Cavallaro A, Luft FC, Uder M, et al. (2015) 23Na magnetic resonance imaging of the lower leg of acute heart failure patients during diuretic treatment. PLoS One 10:e0141336.

Heerspink HJ, Johnsson E, Gause-Nilsson I, Cain VA, and Sjöström CD (2016) Dapagliflozin reduces albuminuria in patients with diabetes and hypertension receiving renin-angiotensin blockers. Diabetes Obes Metab 18:590-597.

Holtkamp FA, de Zeeuw D, Thomas MC, Cooper ME, de Graeff PA, Hillege HJ, Parving HH, Brenner BM, Shahinfar S, and Lambers Heerspink HJ (2011) An acute fall in estimated glomerular filtration rate during treatment with losartan predicts a slower decrease in long-term renal function. Kidney Int 80: 282-287.

Kamei S, Iwamoto M, Kameyama M, Shimoda M, Kinoshita T, Obata A, Kimura T, Hirukawa H, Tatsumi F, Kohara K, et al. (2018) Effect of tofogliflozin on body composition and glycemic control in Japanese subjects with type 2 diabetes mellitus. J Diabetes Res 2018:6470137.

Kohan DE, Fioretto P, Tang W, and List JF (2014) Long-term study of patients with type 2 diabetes and moderate renal impairment shows that dapagliflozin reduces weight and blood pressure but does not improve glycemic control. Kidney Int $\mathbf{8 5}$ 962-971.

Lambers Heerspink HJ, de Zeeuw D, Wie L, Leslie B, and List J (2013) Dapagliflozin a glucose-regulating drug with diuretic properties in subjects with type 2 diabetes. Diabetes Obes Metab 15:853-862.

Lazzara MJ and Deen WM (2007) Model of albumin reabsorption in the proximal tubule. Am J Physiol Renal Physiol 292:F430-F439.

Levine DZ, Iacovitti M, and Robertson SJ (2008) Modulation of single-nephron GFR in the $\mathrm{db} / \mathrm{db}$ mouse model of type 2 diabetes mellitus. II. Effects of renal mass reduction. Am J Physiol Regul Integr Comp Physiol 294:R1840-R1846.

Lewis EJ, Hunsicker LG, Clarke WR, Berl T, Pohl MA, Lewis JB, Ritz E, Atkins RC, Rohde R, and Raz I; Collaborative Study Group (2001) Renoprotective effect of the angiotensin-receptor antagonist irbesartan in patients with nephropathy due to type 2 diabetes. $N$ Engl $J$ Med 345:851-860.

List JF, Woo V, Morales E, Tang W, and Fiedorek FT (2009) Sodium-glucose cotransport inhibition with dapagliflozin in type 2 diabetes. Diabetes Care 32 650-657.

Mahato HS, Ahlstrom C, Jansson-Löfmark R, Johansson U, Helmlinger G, and Hallow KM (2018) Mathematical model of hemodynamic mechanisms and consequences of glomerular hypertension in diabetic mice. NPJ Syst Biol Appl $5: 2$.

Mann JF, Schmieder RE, McQueen M, Dyal L, Schumacher H, Pogue J, Wang X, Maggioni A, Budaj A, Chaithiraphan S, et al.; ONTARGET investigators (2008) Renal outcomes with telmisartan, ramipril, or both, in people at high vascular risk (the ONTARGET study): a multicentre, randomised, double-blind, controlled trial. Lancet 372:547-553.

Marre M, Puig JG, Kokot F, Fernandez M, Jermendy G, Opie L, Moyseev V, Scheen A, Ionescu-Tirgoviste C, Saldanha MH, et al. (2004) Equivalence of indapamide SR and enalapril on microalbuminuria reduction in hypertensive patients with type 2 diabetes: the NESTOR Study. J Hypertens 22:1613-1622.

Maruyama T, Takashima $\mathrm{H}$, Oguma $\mathrm{H}$, Nakamura $\mathrm{Y}$, Ohno $\mathrm{M}$, Utsunomiya $\mathrm{K}$ Furukawa T, Tei R, and Abe M (2019) Canagliflozin improves erythropoiesis in diabetes patients with anemia of chronic kidney disease. Diabetes Technol Ther $\mathbf{2 1}$ $713-720$.

McMurray JJV, Solomon SD, Inzucchi SE, Køber L, Kosiborod MN, Martinez FA, Ponikowski P, Sabatine MS, Anand IS, Bělohlávek J, et al.; DAPA-HF Trial Committees and Investigators (2019) Dapagliflozin in patients with heart failure and reduced ejection fraction. N Engl J Med 381:1995-2008.

Mikami Y, Takagi K, Itaya Y, Ono Y, Matsumura H, Takai Y, and Seki H (2014) Postpartum recovery course in patients with gestational hypertension and preeclampsia. J Obstet Gynaecol Res 40:919-925.

Mosenzon O, Wiviott SD, Cahn A, Rozenberg A, Yanuv I, Goodrich EL, Murphy SA, Heerspink HJL, Zelniker TA, Dwyer JP, et al. (2019) Effects of dapagliflozin on development and progression of kidney disease in patients with type 2 diabetes: an analysis from the DECLARE-TIMI 58 randomised trial. Lancet Diabetes Endocrinol 7:606-617.

Ohara K, Masuda T, Murakami T, Imai T, Yoshizawa H, Nakagawa S, Okada M, Miki A, Myoga A, Sugase T, et al. (2019) Effects of the sodium-glucose cotransporter 2 inhibitor dapagliflozin on fluid distribution: a comparison study with furosemide and tolvaptan. Nephrology (Carlton) 24:904-911. 
Parving HH, Persson F, Lewis JB, Lewis EJ, and Hollenberg NK; AVOID Study Investigators (2008) Aliskiren combined with losartan in type 2 diabetes and nephropathy. N Engl J Med 358:2433-2446.

Pessoa TD, Campos LC, Carraro-Lacroix L, Girardi AC, and Malnic G (2014) Functional role of glucose metabolism, osmotic stress, and sodium-glucose cotransporter isoform-mediated transport on $\mathrm{Na}+\mathrm{H}+$ exchanger isoform 3 activity in the renal proximal tubule. J Am Soc Nephrol 25:2028-2039.

Petrykiv S, Sjöström CD, Greasley PJ, Xu J, Persson F, and Heerspink HJL (2017) Differential effects of dapagliflozin on cardiovascular risk factors at varying degrees of renal function. Clin J Am Soc Nephrol 12:751-759.

Titze J (2009) Water-free sodium accumulation. Semin Dial 22:253-255.

Titze J (2014) Sodium balance is not just a renal affair. Curr Opin Nephrol Hypertens 23:101-105.

Vallon V and Thomson SC (2017) Targeting renal glucose reabsorption to treat hyperglycaemia: the pleiotropic effects of SGLT2 inhibition. Diabetologia 60: $215-225$.

van Bommel EJM, Muskiet MHA, van Baar MJB, Tonneijck L, Smits MM, Emanuel AL, Bozovic A, Danser AHJ, Geurts F, Hoorn EJ, et al. (2020) The renal hemodynamic effects of the SGLT2 inhibitor dapagliflozin are caused by postglomerular vasodilatation rather than pre-glomerular vasoconstriction in metformin-treated patients with type 2 diabetes in the randomized, double-blind RED trial [published correction appears in Kidney Int (2020) 97:1061]. Kidney Int 97:202-212.

Wada Y, Hamamoto Y, Nakatani Y, Fujikawa J, Iwasaki Y, Yoshiji S, Aizawa-Abe M, Iwasaki K, Honjo S, and Hamasaki A (2019) 2343-PUB: reduction of HbA1c after SGLT2 inhibitors correlate with change in plasma osmolarity but not with elevation of hematocrit in Japanese patients with type 2 diabetes. Diabetes 68
Wang W, Hallow KM, and James DA (2016) A tutorial on RxODE: simulating differential equation pharmacometric models in R. CPT Pharmacometrics Syst Pharmacol 5:3-10.

Wanner C, Inzucchi SE, Lachin JM, Fitchett D, von Eynatten M, Mattheus M, Johansen OE, Woerle HJ, Broedl UC, and Zinman B; EMPA-REG OUTCOME Investigators (2016) Empagliflozin and progression of kidney disease in type 2 diabetes. N Engl J Med 375:323-334.

Wilding JP, Norwood P, T'joen C, Bastien A, List JF, and Fiedorek FT (2009) A study of dapagliflozin in patients with type 2 diabetes receiving high doses of insulin plus insulin sensitizers: applicability of a novel insulin-independent treatment. Diabetes Care 32:1656-1662.

Yamout H, Perkovic V, Davies M, Woo V, de Zeeuw D, Mayer C, Vijapurkar U, Kline I, Usiskin K, Meininger G, et al. (2014) Efficacy and safety of canagliflozin in patients with type 2 diabetes and stage 3 nephropathy. Am J Nephrol 40:64-74.

Yavin Y, Mansfield TA, Ptaszynska A, Johnsson K, Parikh S, and Johnsson E (2016) Effect of the SGLT2 inhibitor dapagliflozin on potassium levels in patients with type 2 diabetes mellitus: a pooled analysis. Diabetes Ther 7:125-137.

Zinman B, Wanner C, Lachin JM, Fitchett D, Bluhmki E, Hantel S, Mattheus M, Devins T, Johansen OE, Woerle HJ, et al.; EMPA-REG OUTCOME Investigators (2015) Empagliflozin, cardiovascular outcomes, and mortality in type 2 diabetes. N Engl J Med 373:2117-2128.

Address correspondence to: K. Melissa Hallow, Department of Chemical, Materials, and Biomedical Engineering, University of Georgia, 597 D.W. Brooks Dr., Athens, GA 30602. E-mail: Hallowkm@uga.edu 